\title{
ELEMENTARY NOTIONS OF LATTICE TRIGONOMETRY
}

\author{
OLEG KARPENKOV*
}

\section{Introduction}

\subsection{The goals of this paper and some background}

Consider a two-dimensional oriented real vector space and fix some full-rank lattice in it. A triangle or a polygon is said to be lattice if all its vertices belong to the lattice. The angles of any lattice triangle are said to be lattice.

In this paper we introduce and study lattice trigonometric functions of lattice angles. The lattice trigonometric functions are invariant under the action of the group of lattice-affine transformations (i.e. affine transformations preserving the lattice), like the ordinary trigonometric functions are invariant under the action of the group of Euclidean length preserving transformations of Euclidean space.

One of the initial goals of the present article is to make a complete description of lattice triangles up to the lattice-affine equivalence relation (see Theorem 2.2). The classification problem of convex lattice polygons becomes now classical. There is still no a good description of convex polygons. It is only known that the number of such polygons with lattice area bounded from above by $n$ growths exponentially in $n^{1 / 3}$, while $n$ tends to infinity (see the works of V. Arnold [2], and of I. Bárány and A. M. Vershik [3]).

We extend the geometric interpretation of ordinary continued fractions to define lattice sums of lattice angles and to establish relations on lattice tangents of lattice angles. Further, we describe lattice triangles in terms of lattice sums of lattice angles.

In present paper we also show a lattice version of the sine formula and introduce a relation between the lattice tangents for angles of lattice triangles and the numbers of lattice points on the edges of triangles (see Theorem 1.15).

* Partially supported by NWO-RFBR 047.011.2004.026 (RFBR 05-02-89000-NWO_a) grant, by RFBR SS-1972.2003.1 grant, by RFBR 05-01-02805-CNRSL_a grant, and by RFBR grant 05-01-01012a.

Received October 17, 2006. 
We conclude the paper with applications to toric varieties and some unsolved problems.

The study of lattice angles is an essential part of modern lattice geometry. Invariants of lattice angles are used in the study of lattice convex polygons and polytopes. Such polygons and polytopes play the principal role in Klein's theory of multidimensional continued fractions (see, for example, the works of F. Klein [14], V. I. Arnold [1], E. Korkina [16], M. Kontsevich and Yu. Suhov [15], G. Lachaud [17], and the author [10]).

Lattice polygons and polytopes of the lattice geometry are in the limelight of complex projective toric varieties (see for more information the works of V. I. Danilov [4], G. Ewald [5], T. Oda [18], and W. Fulton [6]). To illustrate, we deduce (in Appendix A) from Theorem 2.2 the corresponding global relations on the toric singularities for projective toric varieties associated to integerlattice triangles. We also show the following simple fact: for any collection with multiplicities of complex-two-dimensional toric algebraic singularities there exists a complex-two-dimensional toric projective variety with the given collection of toric singularities (this result seems to be classical, but it is missing in the literature).

The studies of lattice angles and measures related to them were started by A. G. Khovanskii, A. Pukhlikov in [12] and [13] in 1992. They introduced and investigated special additive polynomial measure for the extended notion of polytopes. The relations between sum-formulas of lattice trigonometric functions and lattice angles in Khovanskii-Pukhlikov sense are unknown to the author.

\subsection{Some distinctions between lattice and Euclidean cases}

Lattice trigonometric functions and Euclidean trigonometric functions have much in common. For example, the values of lattice tangents and Euclidean tangents coincide in a special natural system of coordinates. Nevertheless, lattice geometry differs a lot from Euclidean geometry. We show this with the following four examples.

1 . The angles $\angle A B C$ and $\angle C B A$ are always congruent in Euclidean geometry, but not necessary lattice-congruent in lattice geometry.

2. In Euclidean geometry for any $n \geq 3$ there exist a regular polygon with $n$ vertices, and any two regular polygons with the same number of vertices are homothetic to each other. In lattice geometry there are only six non-homothetic regular lattice polygons: two triangles (distinguished by lattice tangents of angles), two quadrangles, and two octagons. (See a more detailed description in [11].)

3. In Appendix B we will consider three natural criteria for triangle congruence in Euclidean geometry. Only the first criterion can be taken to the case of 
lattice geometry. The others two are false in lattice trigonometry. (We refer to Appendix B.)

4. There exist two non-congruent right angles in lattice geometry. (See Corollary 1.12.)

\subsection{Description of the paper}

This paper is organized as follows.

We start in Section 1 with some general notation of lattice geometry. We define ordinary lattice angles, and the functions lattice sine, tangent, and cosine on the set of ordinary lattice angles, and lattice arctangent for rationals greater than or equal 1. Further we indicate their basic properties. We proceed with the geometrical interpretation of lattice tangents in terms of ordinary continued fractions. In conclusion of Section 1 we study the basic properties of angles in lattice triangles.

In Section 2 we introduce the sum formula for the lattice tangents of ordinary lattice angles of lattice triangles. The sum formula is a lattice generalization of the following Euclidean statement: three angles are the angles of some triangle iff their sum equals $\pi$.

Further in Section 3 we introduce the notion of extended lattice angles and their normal forms and give the definition of sums of extended and ordinary lattice angles. Here we extend the notion of sails in the sense of Klein: we define and study oriented broken lines at unit distance from lattice points.

In Section 4 we finally prove the first statement of the theorem on sums of lattice tangents for angles in lattice triangles. In this section we also describe some relations between continued fractions for lattice oriented broken lines and the lattice tangents for the corresponding extended lattice angles. Further we give a necessary and sufficient condition for an ordered $n$-tuple of angles to be the angles of some convex lattice polygon.

We conclude this paper with three appendices. In Appendix A we describe applications to theory of complex projective toric varieties mentioned above. Further in Appendix B we formulate criterions of lattice congruence for lattice triangles. Finally in Appendix $\mathrm{C}$ we give a list of unsolved problems and questions.

ACKnOwledgement. The author is grateful to V. I. Arnold for constant attention to this work, I. Bárány, A. G. Khovanskii, V. M. Kharlamov, J.M. Kantor, D. Zvonkine, and D. Panov for useful remarks and discussions, and Université Paris-Dauphine - CEREMADE for the hospitality and excellent working conditions. 


\section{Definitions and elementary properties of lattice trigonometric functions}

\subsection{Preliminary notions and definitions}

By $\operatorname{gcd}\left(n_{1}, \ldots, n_{k}\right)$ and by $\operatorname{lcm}\left(n_{1}, \ldots, n_{k}\right)$ we denote the greater common divisor and the less common multiple of the nonzero integers $n_{1}, \ldots, n_{k}$ respectively. Suppose that $a, b$ be arbitrary integers, and $c$ be an arbitrary positive integer. We write that $a \equiv b(\bmod c)$ if the reminders of $a$ and $b$ modulo $c$ coincide.

1.1.1. Lattice notation. Here we define the main objects of lattice geometry, their lattice characteristics, and the relation of $\mathscr{L}$-congruence (lattice-congruence).

Consider $\mathrm{R}^{2}$ and fix some orientation and some lattice in it. A straight line is said to be lattice if it contains at least two distinct lattice points. A ray is said to be lattice if its vertex is a lattice point, and it contains lattice points distinct from its vertex. An angle (i.e. the union of two rays with the common vertex) is said to be ordinary lattice (or just ordinary for short) if the rays defining it are lattice. A segment is called lattice if its endpoints are lattice points.

By a convex polygon we mean a convex hulls of a finite number of points that do not lie in a straight line. A straight line $\pi$ is said to be supporting a convex polygon $P$, if the intersections of $P$ and $\pi$ is not empty, and the whole polygon $P$ is contained in one of the closed half-planes bounded by $\pi$. An intersection of a polygon $P$ with its supporting straight line is called a vertex or an edge of the polygon if the dimension of intersection is zero, or one respectively.

A triangle (or convex polygon) is said to be lattice if all its vertices are lattice points. A lattice triangle is said to be simple if the vectors corresponding to its edges generate the lattice.

The affine transformation is called $\mathscr{L}$-affine if it preserves the set of all lattice points. Consider two arbitrary (not necessary lattice in the above sense) sets. We say that these two sets are $\mathscr{L}$-congruent to each other if there exist a $\mathscr{L}$-affine transformation of $\mathrm{R}^{2}$ taking the first set to the second.

Definition 1.1. The lattice length of a lattice segment $A B$ is the ratio between the Euclidean length of $A B$ and the length of the basic lattice vector for the straight line containing this segment. We denote the lattice length by $1 \ell(A B)$.

By the (non-oriented) lattice area of the convex polygon $P$ we will call the ratio of the Euclidean area of the polygon and the area of any lattice simple triangle, and denote it by $1 \mathrm{~S}(P)$. 
Two lattice segments are $\mathscr{L}$-congruent iff they have equal lattice lengths. The lattice area of the convex polygon is well-defined and is proportional to the Euclidean area of the polygon.

1.1.2. Finite ordinary continued fractions. For any finite sequence $\left(a_{0}, a_{1}\right.$, $\left.\ldots, a_{n}\right)$ where the elements $a_{1}, \ldots, a_{n}$ are positive integers and $a_{0}$ is an arbitrary integer we associate the following rational number $q$ :

$$
q=a_{0}+\frac{1}{a_{1}+\frac{1}{\ddots \quad} \quad \vdots} .
$$

This representation of the rational $q$ is called an ordinary continued fraction for $q$ and denoted by $\left[a_{0}, a_{1}, \ldots, a_{n}\right]$.

An ordinary continued fraction $\left[a_{0}, a_{1}, \ldots, a_{n}\right]$ is said to be odd if $n+1$ is odd, and even if $n+1$ is even. Note that if $a_{n} \neq 1$ then $\left[a_{0}, a_{1}, \ldots, a_{n}\right]=$ $\left[a_{0}, a_{1}, \ldots, a_{n}-1,1\right]$. Let us formulate the following classical theorem.

THEOREM 1.2. For any rational there exist exactly one odd ordinary continued fraction and exactly one even ordinary continued fraction.

\subsection{Definition of lattice trigonometric functions}

In this subsection we define the functions lattice sine, tangent, and cosine on the set of ordinary lattice angles and formulate their basic properties. We describe a geometric interpretation of lattice trigonometric functions in terms of ordinary continued fractions. Then we give the definitions of ordinary angles that are adjacent, transpose, and opposite interior to the given angles. We use the notions of adjacent and transpose ordinary angles to define ordinary lattice right angles.

Let $A, O$, and $B$ be three lattice points that do not lie in the same straight line. We denote the ordinary angle with the vertex at $O$ and the rays $O A$ and $O B$ by $\angle A O B$.

One can chose any other lattice point $C$ in the open lattice ray $O A$ and any lattice point $D$ in the open lattice ray $O B$. For us the angle $\angle A O B$ coincides with $\angle C O D$. We denote this by $\angle A O B=\angle C O D$.

Definition 1.3. Two ordinary angles $\angle A O B$ and $\angle A^{\prime} O^{\prime} B^{\prime}$ are said to be $\mathscr{L}$-congruent if there exist a $\mathscr{L}$-affine transformation that takes the point $O$ to $O^{\prime}$ and the rays $O A$ and $O B$ to the rays $O^{\prime} A^{\prime}$ and $O^{\prime} B^{\prime}$ respectively. We denote this as follows: $\angle A O B \cong \angle A^{\prime} O^{\prime} B^{\prime}$. 
Here we note that the relation $\angle A O B \cong \angle B O A$ holds only for special ordinary angles. (See also below in Subsubsection 1.2.4.)

1.2.1. Definition of lattice sine, tangent, and cosine for an ordinary lattice angle. Consider an arbitrary ordinary angle $\angle A O B$. Let us associate a special basis to this angle. Denote by $\bar{v}_{1}$ and by $\bar{v}_{2}$ the lattice vectors generating the rays of the angle:

$$
\bar{v}_{1}=\frac{\overline{O A}}{1 \ell(O A)}, \quad \text { and } \quad \bar{v}_{2}=\frac{\overline{O B}}{1 \ell(O B)} .
$$

The set of lattice points at unit lattice distance from the lattice straight line $O A$ coincides with the set of all lattice points of two lattice straight lines parallel to $O A$. Since the vectors $\bar{v}_{1}$ and $\bar{v}_{2}$ are linearly independent, the ray $O B$ intersects exactly one of the above two lattice straight lines. Denote this straight line by $l$. The intersection point of the ray $O B$ with the straight line $l$ divides $l$ into two parts. Choose one of the parts which lies in the complement to the convex hull of the union of the rays $O A$ and $O B$, and denote by $D$ the lattice point closest to the intersection of the ray $O B$ with the straight line $l$ (see Figure 1).

Now we choose the vectors $\bar{e}_{1}=\bar{v}_{1}$ and $\bar{e}_{2}=\overline{O D}$. These two vectors are linearly independent and generate the lattice. The basis $\left(\bar{e}_{1}, \bar{e}_{2}\right)$ is said to be associated to the angle $\angle A O B$.

Since $\left(\bar{e}_{1}, \bar{e}_{2}\right)$ is a basis, the vector $\bar{v}_{2}$ has a unique representation of the form:

$$
\bar{v}_{2}=x_{1} \bar{e}_{1}+x_{2} \bar{e}_{2},
$$

where $x_{1}$ and $x_{2}$ are some integers.

DEFINITION 1.4. In the above notation, the coordinates $x_{2}$ and $x_{1}$ are said to be the lattice sine and the lattice cosine of the ordinary angle $\angle A O B$ respectively. The ratio of the lattice sine and the lattice cosine $\left(x_{2} / x_{1}\right)$ is said to be the lattice tangent of $\angle A O B$.

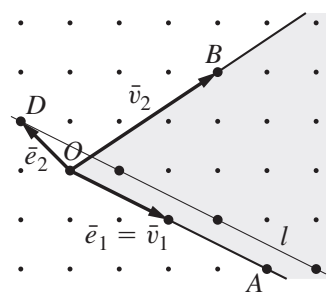

$$
\begin{aligned}
& \bar{v}_{2}=5 \bar{e}_{1}+7 \bar{e}_{2} \\
& 1 \sin \angle A O B=7 \\
& 1 \cos \angle A O B=5 \\
& 1 \tan \angle A O B=7 / 5
\end{aligned}
$$

FIGURE 1 . An ordinary angle $\angle A O B$ and its lattice trigonometric functions. 
Figure 1 shows an example of lattice angle with the lattice sine equals 7 and the lattice cosine equals 5 .

Let us briefly enumerate some elementary properties of lattice trigonometric functions.

Proposition 1.5. a) The lattice sine and cosine of any ordinary angle are relatively-prime positive integers.

b) The values of lattice trigonometric functions for $\mathscr{L}$-congruent ordinary angles coincide.

c) The lattice sine of an ordinary angle coincide with the index of the sublattice generated by all lattice vectors of two angle rays in the lattice.

d) For any ordinary angle $\alpha$ the following inequalities hold:

$$
1 \sin \alpha \geq \operatorname{lcos} \alpha, \quad \text { and } \quad \operatorname{ltan} \alpha \geq 1 .
$$

The equalities hold iff the lattice vectors of the angle rays generate the whole lattice.

e) (Description of lattice angles) Two ordinary angles $\alpha$ and $\beta$ are $\mathscr{L}$ congruent iff $\operatorname{ltan} \alpha=1 \tan \beta$.

1.2.2. Lattice arctangent. Let us fix the origin $O$ and a lattice basis $\bar{e}_{1}$ and $\bar{e}_{2}$.

Definition 1.6. Consider an arbitrary rational $p \geq 1$. Let $p=m / n$, where $m$ and $n$ are positive integers. Suppose $A=O+\bar{e}_{1}$, and $B=O+n \bar{e}_{1}+m \bar{e}_{2}$. The ordinary angle $\angle A O B$ is said to be the arctangent of $p$ in the fixed basis and denoted by $\operatorname{larctan}(p)$.

The invariance of lattice tangents immediately implies the following properties.

Proposition 1.7. a) For any rational $s \geq 1$, we have: $\operatorname{ltan}(\operatorname{larctan} s)=s$.

b) For any ordinary angle $\alpha$ the following holds: $\operatorname{larctan}(\tan \alpha) \cong \alpha$.

1.2.3. Lattice tangents, length-sine sequences, sails, and continued fractions. Let us start with the notion of sails for the ordinary angles. This notion is taken from theory of multidimensional continued fractions in the sense of Klein (see, for example, the works of F. Klein [14], and V. Arnold [1]).

Consider an ordinary angle $\angle A O B$. Let also the vectors $\overline{O A}$ and $\overline{O B}$ be linearly independent, and of unit lattice length. Denote the closed convex solid cone for the ordinary angle $\angle A O B$ by $C(A O B)$. The boundary of the convex hull of all lattice points of the cone $C(A O B)$ except the origin is homeomorphic to the straight line. This boundary contains the points $A$ and $B$. The closed part of this boundary contained between the points $A$ and $B$ is called the sail for the cone $C(A O B)$. 
A lattice point of the sail is said to be a vertex of the sail if there is no lattice segment of the sail containing this point in the interior. The sail of the cone $C(A O B)$ is a broken line with a finite number of vertices and without self intersections. Let us orient the sail in the direction from $A$ to $B$, and denote the vertices of the sail by $V_{i}$ (for $0 \leq i \leq n$ ) according to the orientation of the sail (such that $V_{0}=A$, and $V_{n}=B$ ).

Definition 1.8. Let the vectors $\overline{O A}$ and $\overline{O B}$ of the ordinary angle $\angle A O B$ be linearly independent, and of unit lattice length. Let $V_{i}$, where $0 \leq i \leq n$, be the vertices of the corresponding sail. The sequence of lattice lengths and sines

(l $\ell\left(V_{0} V_{1}\right), 1 \sin \angle V_{0} V_{1} V_{2}, l \ell\left(V_{1} V_{2}\right), 1 \sin \angle V_{1} V_{2} V_{3}$,

$$
\left.\ldots, 1 \ell\left(V_{n-2} V_{n-1}\right), 1 \sin \angle V_{n-2} V_{n-1} V_{n}, 1 \ell\left(V_{n-1} V_{n}\right)\right)
$$

is called the lattice length-sine sequence for the ordinary angle $\angle A O B$. Further we say $L L S$-sequence for short.

Remark 1.9. The elements of the lattice LLS-sequence for any ordinary angle are positive integers. The LLS-sequences of $\mathscr{L}$-congruent ordinary angles coincide.

THEOREM 1.10. Let $\left(a_{0}, a_{1}, \ldots, a_{2 n-3}, a_{2 n-2}\right)$ be the LLS-sequence for the ordinary angle $\angle A O B$. Then the lattice tangent of the ordinary angle $\angle A O B$ equals to the value of the following ordinary continued fraction

$$
\left[a_{0}, a_{1}, \ldots, a_{2 n-3}, a_{2 n-2}\right] .
$$

On Figure 2 we show an example of an ordinary angle with tangent equivalent to $7 / 5$.
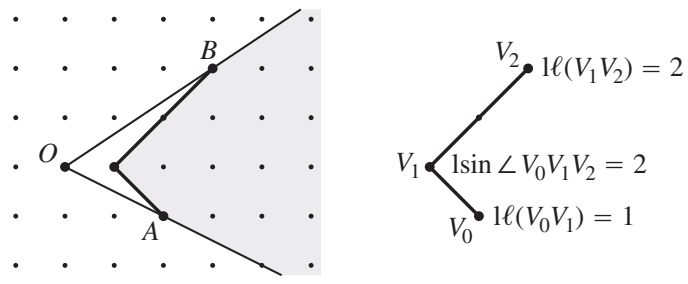

Figure 2. $\operatorname{ltan} \angle A O B=\frac{7}{5}=1+\frac{1}{2+1 / 2}$.

Further in Theorem 3.5 we formulate and prove a general statement for generalized sails and signed lattice length-sine sequences. In the proof of Theorem 3.5 we refer only on the preceding statements and definitions of Subsection 3.1, 
that are independent of the statements and theorems of all previous sections. For these reasons we skip now the proof of Theorem 1.10 (see also Remark 3.6).

1.2.4. Adjacent, transpose, and opposite interior ordinary angles. An ordinary angle $\angle B O A$ is said to be transpose to the ordinary angle $\angle A O B$. We denote it by $(\angle A O B)^{t}$. An ordinary angle $\angle B O A^{\prime}$ is said to be adjacent to an ordinary angle $\angle A O B$ if the points $A, O$, and $A^{\prime}$ are contained in the same straight line, and the point $O$ lies between $A$ and $A^{\prime}$. We denote the ordinary angle $\angle B O A^{\prime}$ by $\pi-\angle A O B$. The ordinary angle is said to be right if it is $\mathscr{L}$-congruent to the adjacent and to the transpose ordinary angles.

It immediately follows from the definition, that for any ordinary angle $\alpha$ the angles $\left(\alpha^{t}\right)^{t}$ and $\pi-(\pi-\alpha)$ are $\mathscr{L}$-congruent to $\alpha$.

In the next theorem we use the following notion. Suppose that some integers $a, b$ and $c$, where $c \geq 1$, satisfy the following: $a b \equiv 1(\bmod c)$. Then we denote $a \equiv(b(\bmod c))^{-1}$.

THEOREm 1.11. Consider an ordinary angle $\alpha$. If $\alpha \cong \operatorname{larctan}(1)$, then

$$
\alpha^{t} \cong \pi-\alpha \cong \operatorname{larctan}(1) .
$$

Suppose now, that $\alpha ¥ \operatorname{larctan}(1)$, then

$$
\begin{aligned}
& 1 \sin \left(\alpha^{t}\right)=1 \sin \alpha, \quad 1 \cos \left(\alpha^{t}\right) \equiv(\operatorname{lcos} \alpha(\bmod 1 \sin \alpha))^{-1} ; \\
& 1 \sin (\pi-\alpha)=1 \sin \alpha, \quad 1 \cos (\pi-\alpha) \equiv(-\operatorname{lcos} \alpha(\bmod 1 \sin \alpha))^{-1} .
\end{aligned}
$$

Note also, that $\pi-\alpha \cong \operatorname{larctan}^{t}\left(\frac{\operatorname{ltan} \alpha}{\operatorname{ltan}(\alpha)-1}\right)$.

Theorem 1.11 (after applying Theorem 1.10) immediately reduces to the theorem of P. Popescu-Pampu. We refer the readers to his work [19] for the proofs.

1.2.5. Right ordinary lattice angles. It turns out that in lattice geometry there exist exactly two lattice non-equivalent right ordinary angles.

COROLlaRy 1.12. Any ordinary right angle is $\mathscr{L}$-congruent to exactly one of the following two angles: larctan(1), or larctan(2).

Consider two lattice parallel distinct straight lines $A B$ and $C D$, where $A, B$, $C$, and $D$ are lattice points. Let the points $A$ and $D$ be in different open halfplanes with respect to the straight line $B C$. Then the ordinary angle $\angle A B C$ is called opposite interior to the ordinary angle $\angle D C B$. Further we use the following proposition on opposite interior ordinary angles.

PRoposition 1.13. Two opposite interior to each other ordinary angles are $\mathscr{L}$-congruent.

The proof is left for the reader as an exercise. 


\subsection{Basic lattice trigonometry of lattice angles in lattice triangles}

In this subsection we introduce the sine formula for angles and edges of lattice triangles. Further we show how to find the lattice tangents of all angles and the lattice lengths of all edges of any lattice triangle, if the lattice lengths of two edges and the lattice tangent of the angle between them are given.

Let $A, B, C$ be three distinct and not collinear lattice points. We denote the lattice triangle with the vertices $A, B$, and $C$ by $\triangle A B C$. The lattice triangles $\triangle A B C$ and $\triangle A^{\prime} B^{\prime} C^{\prime}$ are said to be $\mathscr{L}$-congruent if there exist a $\mathscr{L}$-affine transformation which takes the point $A$ to $A^{\prime}, B$ to $B^{\prime}$, and $C$ to $C^{\prime}$ respectively. We denote: $\triangle A B C \cong \triangle A^{\prime} B^{\prime} C^{\prime}$.

Proposition 1.14 (The sine formula for lattice triangles). The following holds for any lattice triangle $\triangle A B C$.

$$
\frac{1 \ell(A B)}{1 \sin \angle B C A}=\frac{1 \ell(B C)}{1 \sin \angle C A B}=\frac{1 \ell(C A)}{1 \sin \angle A B C}=\frac{1 \ell(A B) 1 \ell(B C) 1 \ell(C A)}{1 \mathrm{~S}(\triangle A B C)} .
$$

Proof. The statement of Proposition 1.14 follows directly from the definition of lattice sine.

Suppose that we know the lattice lengths of the edges $A B, A C$ and the lattice tangent of $\angle B A C$ in the triangle $\triangle A B C$. Now we show how to restore the lattice length and the lattice tangents for the the remaining edge and ordinary angles of the triangle.

For the simplicity we fix some lattice basis and use the system of coordinates $O X Y$ corresponding to this basis (denoted $(*, *)$ ).

TheOREM 1.15. Consider some triangle $\triangle A B C$. Let

$$
1 \ell(A B)=c, \quad 1 \ell(A C)=b, \quad \text { and } \quad \angle C A B \cong \alpha .
$$

Then the ordinary angles $\angle B C A$ and $\angle A B C$ are defined in the following way.

$$
\begin{aligned}
& \angle B C A \cong \begin{cases}\pi-\operatorname{larctan}\left(\frac{c \operatorname{lin} \alpha}{c \cos \alpha-b}\right) & \text { if } c \cos \alpha>b \\
\arctan (1) & \text { if } c \cos \alpha=b \\
\operatorname{larctan}^{t}\left(\frac{c \operatorname{lin} \alpha}{b-c \cos \alpha}\right) & \text { if } c \cos \alpha<b,\end{cases} \\
& \angle A B C \cong \begin{cases}\left(\pi-\operatorname{larctan}\left(\frac{b \operatorname{lin}\left(\alpha^{t}\right)}{b \cos \left(\alpha^{t}\right)-c}\right)\right)^{t} & \text { if } b \cos \left(\alpha^{t}\right)>c \\
\arctan (1) & \text { if } b \cos \left(\alpha^{t}\right)=c \\
\arctan \left(\frac{b \operatorname{lin}\left(\alpha^{t}\right)}{c-b \cos \left(\alpha^{t}\right)}\right) & \text { if } b \cos \left(\alpha^{t}\right)<c .\end{cases}
\end{aligned}
$$


For the lattice length of the edge $C B$ we have

$$
\frac{1 \ell(C B)}{1 \sin \alpha}=\frac{b}{1 \sin \angle A B C}=\frac{c}{1 \sin \angle B C A} .
$$

Proof. Let $\alpha \cong \operatorname{larctan}(p / q)$, where $\operatorname{gcd}(p, q)=1$. Then $\triangle C A B \cong$ $\triangle D O E$ where $D=(b, 0), O=(0,0)$, and $E=(q c, p c)$. Let us now find the ordinary angle $\angle E D O$. Denote by $Q$ the point $(q c, 0)$. If $q c-b=0$, then $\angle B C A=\angle E D O=\operatorname{larctan} 1$. If $q c-b \neq 0$, then we have

$$
\angle Q D E \cong \operatorname{larctan}\left(\frac{c p}{|c q-b|}\right) \cong \operatorname{larctan}\left(\frac{c \operatorname{lsin} \alpha}{|c \cos \alpha-b|}\right) .
$$

The expression for $\angle B C A$ follows directly from the above expression for $\angle Q D E$, since $\angle B C A \cong \angle Q D E$. (See Figure 3: here $1 \ell(O D)=b, 1 \ell(O Q)=$ $c \operatorname{los} \alpha$, and therefore $\ell \ell(D Q)=|c \operatorname{los} \alpha-b|$.)
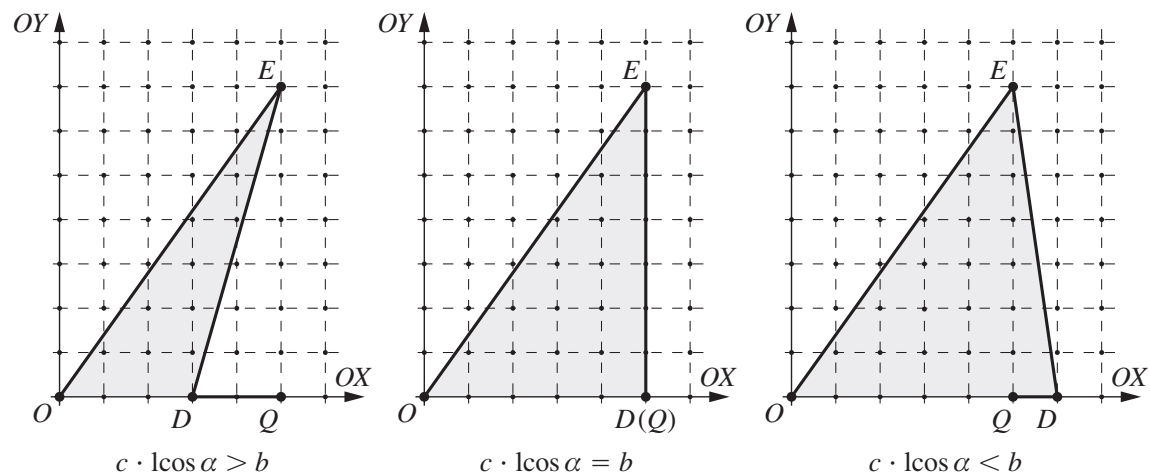

FIGURE 3. Three possible configuration of points $O, D$, and $Q$.

To obtain the expression for $\angle A B C$ we consider the triangle $\triangle B A C$. Calculate $\angle C B A$ and then transpose all ordinary angles in the expression. Since

$$
\begin{aligned}
1 \mathrm{~S}(A B C) & =1 \ell(A B) 1 \ell(A C) \sin \angle C A B \\
& =1 \ell(B A) 1 \ell(B C) 1 \sin \angle B C A \\
& =1 \ell(C B) 1 \ell(C A) 1 \sin \angle A B C,
\end{aligned}
$$

we have the last statement of the theorem. 


\section{Theorem on sum of lattice tangents for the ordinary lattice angles of lattice triangles. Proof of its second statement}

Throughout this section we fix some lattice basis and use the system of coordinates $O X Y$ corresponding to this basis.

\subsection{Finite continued fractions with not necessary positive elements}

We start this section with the notation for finite continued fractions with not necessary positive elements. Let us extend the set of rationals $\mathrm{Q}$ with the operations + and $1 / *$ on it with the element $\infty$. We pose $q \pm \infty=\infty$, $1 / 0=\infty, 1 / \infty=0$ (we do not define $\infty \pm \infty$ here). Denote this extension by $\overline{\mathrm{Q}}$.

For any finite sequence of integers $\left(a_{0}, a_{1}, \ldots, a_{n}\right)$ we associate an element $q$ of $\overline{\mathrm{Q}}$ :

$$
q=a_{0}+\frac{1}{a_{1}+\frac{1}{\ddots \quad} \quad \vdots} .
$$

and denote it by $] a_{0}, a_{1}, \ldots, a_{n}[$.

Let $q_{i}$ be some rationals, $i=1, \ldots, k$. Suppose that the odd continued fraction for $q_{i}$ is $\left[a_{i, 0}, a_{i, 1}, \ldots, a_{i, 2 n_{i}}\right]$ for $i=1, \ldots, k$. We denote by ]$q_{1}, q_{2}, \ldots, q_{n}[$ the following number

$$
] a_{1,0}, a_{1 ; 1}, \ldots, a_{1,2 n_{1}}, a_{2,0}, a_{2,1}, \ldots, a_{2,2 n_{2}}, \ldots a_{k, 0}, a_{k, 1}, \ldots, a_{k, 2 n_{k}}[.
$$

\subsection{Formulation of the theorem and proof of its second statement}

In Euclidean geometry the sum of Euclidean angles of the triangle equals $\pi$. For any 3-tuple of angles with the sum equals $\pi$ there exist a triangle with these angles. Two Euclidean triangles with the same angles are homothetic. Let us show a generalization of these statements to the case of lattice geometry.

Let $n$ be an arbitrary positive integer, and $A=(x, y)$ be an arbitrary lattice point. Denote by $n A$ the point $(n x, n y)$.

Definition 2.1. Consider any convex polygon or broken line with vertices $A_{0}, \ldots, A_{k}$. The polygon or broken line $n A_{0} \ldots n A_{k}$ is called $n$-multiple (or multiple) to the given polygon or broken line.

Theorem 2.2 (On sum of lattice tangents of angles in lattice triangles). a) Let $\left(\alpha_{1}, \alpha_{2}, \alpha_{3}\right)$ be an ordered 3-tuple of ordinary angles. There exists a triangle with three consecutive ordinary angles $\mathscr{L}$-congruent to $\alpha_{1}, \alpha_{2}$, and 
$\alpha_{3}$ iff there exists $i \in\{1,2,3\}$ such that the angles $\alpha=\alpha_{i}, \beta=\alpha_{i+1}(\bmod 3)$, and $\gamma=\alpha_{i+2}(\bmod 3)$ satisfy the following conditions:

i) for $A=] 1 \tan \alpha,-1,1 \tan \beta[$ the following holds $A<0$, or $A>\operatorname{ltan} \alpha$, or $A=\infty$;

ii) $] \tan \alpha,-1,1 \tan \beta,-1,1 \tan \gamma[=0$.

b) Let the consecutive ordinary angles of some triangle be $\alpha, \beta$, and $\gamma$. Then this triangle is multiple to the triangle with vertices $A_{0}=(0,0), B_{0}=$ $\left(\lambda_{2} l \cos \alpha, \lambda_{2} l \sin \alpha\right)$, and $C_{0}=\left(\lambda_{1}, 0\right)$, where

$$
\lambda_{1}=\frac{1 \mathrm{~cm}(1 \sin \alpha, 1 \sin \beta, 1 \sin \gamma)}{\operatorname{gcd}(1 \sin \alpha, 1 \sin \gamma)}, \quad \text { and } \quad \lambda_{2}=\frac{1 \mathrm{~cm}(1 \sin \alpha, 1 \sin \beta, 1 \sin \gamma)}{\operatorname{gcd}(1 \sin \alpha, 1 \sin \beta)} .
$$

Let us say a few words about the essence of the theorem. In Euclidean geometry on the plane the condition on the angles of triangles can be rewritten with tangent functions in the following way. A triangle with angles exists $\alpha$, $\beta$, and $\gamma$ iff $\tan (\alpha+\beta+\gamma)=0$ and $\tan (\alpha+\beta) \notin[0 ; \tan \alpha]$ (here without lose of generality we suppose that $\alpha$ is acute). Theorem 2.2 is a translation of this condition into lattice case.

In addition we say that there is no a good description of lattice polygons terms of lattice invariants at present. Theorem 2.2 gives such description for the case of triangles.

At this moment we do not have the necessary notation to prove the first statement of Theorem 2.2. For a proof we need first to define extended angles and their sums, and study their properties. We give a proof further in Subsections 4.2 and 4.3. We prove the second statement of the theorem below in this subsection.

Remark 2.3. Note that the statement of Theorem 2.2a holds only for odd continued fractions for the tangents of the correspondent angles. We illustrate this with the following example. Consider a lattice triangle with the lattice area equals 7 and all angles $\mathscr{L}$-congruent to $\operatorname{larctan} 7 / 3$. If we take the odd continued fractions $7 / 3=[2,2,1]$ for all lattice angles of the triangle, then we have

$$
] 2,2,1,-1,2,2,1,-1,2,2,1[=0 .
$$

If we take the even continued fractions $7 / 3=[2,3]$ for all angles of the triangle, then we have

$$
] 2,3,-1,2,3,-1,2,3\left[=\frac{35}{13} \neq 0 .\right.
$$


Proof of the Second statement of Theorem 2.2. Consider a triangle $\triangle A B C$ with ordinary angles $\alpha, \beta$, and $\gamma$ (at vertices at $A, B$, and $C$ respectively). Suppose that for any $k>1$ and any lattice triangle $\triangle K L M$ the triangle $\triangle A B C$ is not $\mathscr{L}$-congruent to the $k$-multiple of $\triangle K L M$. In other world, we have

$$
\operatorname{gcd}(\ell \ell(A B), 1 \ell(B C), 1 \ell(C A))=1 \text {. }
$$

Suppose that $S$ is the lattice area of $\triangle A B C$. Then by the sine formula the following holds

$$
\left\{\begin{array}{l}
1 \ell(A B) 1 \ell(A C)=S / 1 \sin \alpha \\
1 \ell(B C) 1 \ell(B A)=S / 1 \sin \beta \\
1 \ell(C A) 1 \ell(C B)=S / 1 \sin \gamma
\end{array} .\right.
$$

Since $\operatorname{gcd}(1 \ell(A B), 1 \ell(B C), 1 \ell(C A))=1$, we have $1 \ell(A B)=\lambda_{1}$ and $1 \ell(A C)=$ $\lambda_{2}$.

Therefore, the lattice triangle $\triangle A B C$ is $\mathscr{L}$-congruent to the lattice triangle $\triangle A_{0} B_{0} C_{0}$ of the theorem.

\section{Extension of ordinary lattice angles. Notion of sums of lattice angles}

Throughout this section we work in with an oriented two-dimensional real vector space and a fixed lattice in it. We again fix some (positively oriented) lattice basis and use the system of coordinates $O X Y$ corresponding to this basis.

The $\mathscr{L}$-affine transformation is said to be proper if it is orientation-preserving (we denote it by $\mathscr{L}_{+}$-affine transformation).

We say that two sets are $\mathscr{L}_{+}$-congruent to each other if there exist a $\mathscr{L}_{+}{ }^{-}$ affine transformation of $\mathrm{R}^{2}$ taking the first set to the second.

\subsection{On a particular generalization of sails in the sense of Klein}

In this subsection we introduce the definition of an oriented broken lines at unit lattice distance from a lattice point. This notion is a direct generalization of the notion of a sail in the sense of Klein (see page 167 for the definition of a sail). We extend the definition of LLS-sequences and continued fractions to the case of these broken lines. We show that extended LLS-sequence for oriented broken lines uniquely identifies the $\mathscr{L}_{+}$-congruence class of the corresponding broken line. Further, we study the geometrical interpretation of the corresponding continued fraction.

3.1.1. Definition of a lattice signed length-sine sequence. Let us extend the definition of LLS-sequence to the case of certain broken lines. 
For the 3-tuples of lattice points $A, B$, and $C$ we define the function sgn as follows:

$$
\operatorname{sgn}(A B C)=\left\{\begin{aligned}
+1, & \begin{array}{l}
\text { if the pair of vectors } \overline{B A} \text { and } \overline{B C} \text { defines the positive } \\
\text { orientation. }
\end{array} \\
0, & \begin{array}{l}
\text { if the points } A, B, \text { and } C \text { are contained in the same } \\
\text { straight line. }
\end{array} \\
-1, & \begin{array}{l}
\text { if the pair of vectors } \overline{B A} \text { and } \overline{B C} \text { defines the negative } \\
\text { orientation. }
\end{array}
\end{aligned}\right.
$$

We also denote by sign $: R \rightarrow\{-1,0,1\}$ the sign function over reals.

A segment $A B$ is said to be at unit distance from the point $C$ if the lattice vectors of the segment $A B$, and the vector $\overline{A C}$ generate the lattice.

A union of (ordered) lattice segments $A_{0} A_{1}, A_{1} A_{2}, \ldots, A_{n-1} A_{n}(n>0)$ is said to be a lattice oriented broken line and denoted by $A_{0} A_{1} A_{2} \ldots A_{n}$ if any two consecutive segments are not contained in the same straight line. We also say that the lattice oriented broken line $A_{n} A_{n-1} A_{n-2} \ldots A_{0}$ is inverse to the lattice oriented broken line $A_{0} A_{1} A_{2} \ldots A_{n}$.

Definition 3.1. Consider a lattice oriented broken line and a lattice point $V$ in the complement to this line. The broken line is said to be at unit distance from the point $V$ (or $V$-broken line for short) if all edges of the broken line are at unit distance from $V$.

Let us now associate to any lattice oriented $V$-broken line for some lattice point $V$ the following sequence of non-zero elements.

Definition 3.2. Let $A_{0} A_{1} \ldots A_{n}$ be a lattice oriented $V$-broken line. The sequence of integers $\left(a_{0}, \ldots, a_{2 n-2}\right)$ defined as follows:

$$
\begin{aligned}
a_{0}= & \operatorname{sgn}\left(A_{0} V A_{1}\right) l \ell\left(A_{0} A_{1}\right), \\
a_{1}= & \operatorname{sgn}\left(A_{0} V A_{1}\right) \operatorname{sgn}\left(A_{1} V A_{2}\right) \operatorname{sgn}\left(A_{0} A_{1} A_{2}\right) \sin \angle A_{0} A_{1} A_{2}, \\
a_{2}= & \operatorname{sgn}\left(A_{1} V A_{2}\right) l \ell\left(A_{1} A_{2}\right), \\
& \ldots \\
a_{2 n-3}= & \operatorname{sgn}\left(A_{n-2} V A_{n-1}\right) \operatorname{sgn}\left(A_{n-1} V A_{n}\right) \\
& \quad \operatorname{sgn}\left(A_{n-2} A_{n-1} A_{n}\right) \sin \angle A_{n-2} A_{n-1} A_{n}, \\
a_{2 n-2}= & \operatorname{sgn}\left(A_{n-1} V A_{n}\right) \operatorname{l} \ell\left(A_{n-1} A_{n}\right),
\end{aligned}
$$

is called an lattice signed length-sine sequence for the lattice oriented $V$-broken line. Further we will say $L S L S$-sequence for short.

The element $] a_{0}, a_{1}, \ldots, a_{2 n-2}$ [ of $\overline{\mathbf{Q}}$ is called the continued fraction for the broken line $A_{0} A_{1} \ldots A_{n}$. 
If we take LSLS-sequence for some broken line which is a sail, than LSLSsequence is exactly LLS-sequence for the corresponding angle. So LSLSsequence is a natural combinatorical-geometrical generalization of LLS-sequences. Note also that if we know the whole LSLS-sequence for some $V$ broken line and the coordinates of points $V, A_{0}$, and $A_{1}$ then the coordinates of $A_{2}, \ldots$ can be restored in the unique way.

Let us show how to identify geometrically the signs of elements of the LSLS-sequence for a lattice oriented $V$-broken line on Figure 4.

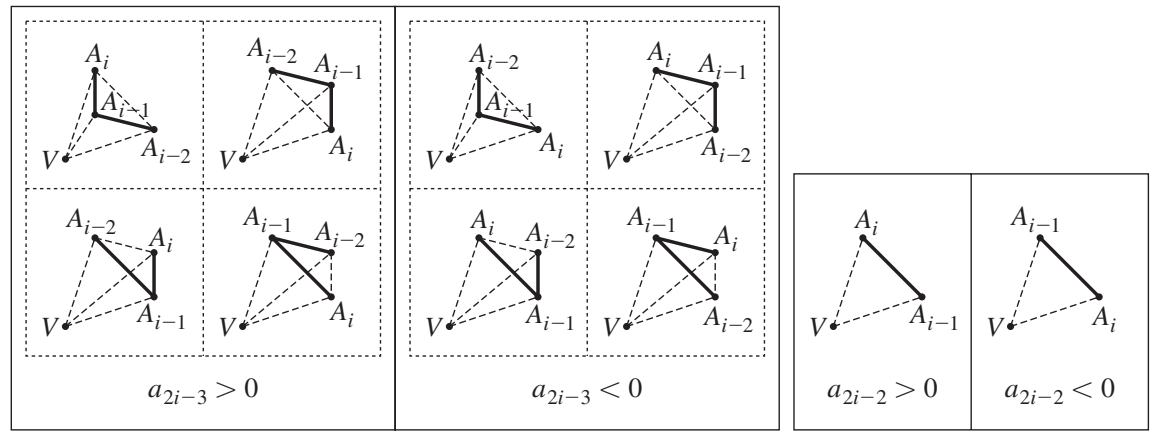

FIGURE 4. All possible (non-degenerate) $\mathscr{L}_{+}$-affine decompositions for angles and segments of a LSLS-sequence.

On Figure 5 we show an example of lattice oriented $V$-broken line and the corresponding LSLS-sequence.

FIGURE 5. A lattice oriented $V$-broken line and the corresponding LSLS-sequence.

Proposition 3.3. A LSLS-sequence for the given lattice oriented broken line and the lattice point is invariant under the group action of the $\mathscr{L}_{+}$-affine transformations.

3.1.2. On $\mathscr{L}_{+}$-congruence of lattice oriented $V$-broken lines. Let us formulate necessary and sufficient conditions for two lattice oriented $V$-broken lines (for the same lattice point $V$ ) to be $\mathscr{L}_{+}$-congruent. 
THEOREM 3.4. The LSLS-sequences of two lattice oriented $V_{1}$-broken and $V_{2}$-broken lines (for two lattice points $V_{1}$ and $V_{2}$ ) coincide iff there exist a $\mathscr{L}_{+}$-affine transformation taking the point $V_{1}$ to $V_{2}$ and one lattice oriented broken line to the other.

Proof. The LSLS-sequence for any lattice oriented $V$-broken line is uniquely defined, and by Proposition 3.3 is invariant under the group action of $\mathscr{L}$ affine orientation preserving transformations. Therefore, the LSLS-sequences for two $\mathscr{L}_{+}$-congruent lattice oriented broken lines coincide.

Suppose now that two lattice oriented $V_{1}$-broken and $V_{2}$-broken lines $A_{0} \ldots A_{n}$, and $B_{0} \ldots B_{n}$ respectively have the same LSLS-sequence $\left(a_{0}, a_{1}\right.$, $\left.\ldots, a_{2 n-3}, a_{2 n-2}\right)$. Let us prove that these broken lines are $\mathscr{L}_{+}$-congruent. Without loose of generality we consider the point $V_{1}$ at the origin $O$.

Let $\xi$ be the $\mathscr{L}_{+}$-affine transformation taking the point $V_{2}$ to the point $V_{1}=O, B_{0}$ to $A_{0}$, and the lattice straight line containing $B_{0} B_{1}$ to the lattice straight line containing $A_{0} A_{1}$. Let us prove inductively that $\xi\left(B_{i}\right)=A_{i}$.

Base of induction. Since $a_{0}=b_{0}$, we have

$$
\operatorname{sgn}\left(A_{0} O A_{1}\right) 1 \ell\left(A_{0} A_{1}\right)=\operatorname{sgn}\left(\xi\left(B_{0}\right) O \xi\left(B_{1}\right)\right) 1 \ell\left(\xi\left(B_{0}\right) \xi\left(B_{1}\right)\right) .
$$

Thus, the lattice segments $A_{0} A_{1}$ and $A_{0} \xi\left(B_{1}\right)$ are of the same lattice length and of the same direction. Therefore, $\xi\left(B_{1}\right)=A_{1}$.

Step of induction. Suppose, that $\xi\left(B_{i}\right)=A_{i}$ holds for any nonnegative integer $i \leq k$, where $k \geq 1$. Let us prove, that $\xi\left(B_{k+1}\right)=A_{k+1}$. Denote by $C_{k+1}$ the lattice point $\xi\left(B_{k+1}\right)$. Let $A_{k}=\left(q_{k}, p_{k}\right)$. Denote by $A_{k}^{\prime}$ the closest lattice point of the segment $A_{k-1} A_{k}$ to the vertex $A_{k}$. Suppose that $A_{k}^{\prime}=\left(q_{k}^{\prime}, p_{k}^{\prime}\right)$. We know also

$$
\begin{aligned}
a_{2 k-1} & =\operatorname{sgn}\left(A_{k-1} O A_{k}\right) \operatorname{sgn}\left(A_{k} O C_{k+1}\right) \operatorname{sgn}\left(A_{k-1} A_{k} C_{k+1}\right) \operatorname{lin} \angle A_{k-1} A_{k} C_{k+1}, \\
a_{2 k} & =\operatorname{sgn}\left(A_{k} O C_{k+1}\right) \operatorname{l}\left(A_{k} C_{k+1}\right) .
\end{aligned}
$$

Let the coordinates of $C_{k+1}$ be $(x, y)$. Since $1 \ell\left(A_{k} C_{k+1}\right)=\left|a_{2 k}\right|$ and the segment $A_{k} C_{k+1}$ is at unit distance to the origin $O$, we have $\operatorname{lS}\left(\triangle O A_{k} C_{k+1}\right)=$ $\left|a_{2 k}\right|$. Since the segment $O A_{k}$ is of the unit lattice length, the coordinates of $C_{k+1}$ satisfy the following equation:

$$
\left|-p_{k} x+q_{k} y\right|=\left|a_{2 k}\right| .
$$

Since $\operatorname{sgn}\left(A_{k} O C_{k+1}\right) l \ell\left(A_{k} C_{k+1}\right)=\operatorname{sign}\left(a_{2 k}\right)$, we have $-p_{k} x+q_{k} y=a_{2 k}$.

Since lsin $\angle A_{k}^{\prime} A_{k} C_{k+1}=1 \sin \angle A_{k-1} A_{k} C_{k+1}=\left|a_{2 k-1}\right|$, and the lattice lengths of $A_{k} C_{k+1}$, and $A_{k}^{\prime} A_{k}$ are $\left|a_{2 k}\right|$ and 1 respectively, we have $\operatorname{lS}\left(\triangle A_{k}^{\prime} A_{k} C_{k+1}\right)=$ $\left|a_{2 k-1} a_{2 k}\right|$. Therefore, the coordinates of $C_{k+1}$ satisfy the following equation:

$$
\left|-\left(p_{k}-p_{k}^{\prime}\right)\left(x-q_{k}\right)+\left(q_{k}-q_{k}^{\prime}\right)\left(y-p_{k}\right)\right|=\left|a_{2 k-1} a_{2 k}\right| .
$$


Since

$$
\left\{\begin{aligned}
\operatorname{sgn}\left(A_{k-1} O A_{k}\right) \operatorname{sgn}\left(A_{k} O C_{k+1}\right) \operatorname{sgn}\left(A_{k-1} A_{k} C_{k+1}\right) & =\operatorname{sign}\left(a_{2 k-1}\right) \\
\operatorname{sgn}\left(A_{k} O C_{k+1}\right) & =\operatorname{sign}\left(a_{2 k}\right)
\end{aligned}\right.
$$

we have $\left(p_{k}-p_{k}^{\prime}\right)\left(x-q_{k}\right)-\left(q_{k}-q_{k}^{\prime}\right)\left(y-p_{k}\right)=\operatorname{sgn}\left(A_{k-1} O A_{k}\right) a_{2 k-1} a_{2 k}$.

We obtain the following:

$$
\left\{\begin{array}{rl}
-p_{k} x+q_{k} y & =a_{2 k} \\
\left(p_{k}-p_{k}^{\prime}\right)\left(x-q_{k}\right)-\left(q_{k}-q_{k}^{\prime}\right)\left(y-p_{k}\right) & =\operatorname{sgn}\left(A_{k-1} O A_{k}\right) a_{2 k-1} a_{2 k}
\end{array} .\right.
$$

Since

$$
\left|\operatorname{det}\left(\begin{array}{cc}
-p_{k} & q_{k} \\
p_{k}^{\prime}-p_{k} & q_{k}-q_{k}^{\prime}
\end{array}\right)\right|=1,
$$

there exist a unique integer solution for the system of equations for $x$ and $y$. Hence, the points $A_{k+1}$ and $C_{k+1}$ have the same coordinates. Therefore, $\xi\left(B_{k+1}\right)=A_{k+1}$. We have proven the step of induction.

The proof of Theorem 3.4 is completed by induction.

3.1.3. Values of continued fractions for lattice oriented broken lines at unit distance from the origin. Now we show the relation between lattice oriented broken lines at unit distance from the origin $O$ and the corresponding continued fractions for them.

THEOREM 3.5. Let $A_{0} A_{1} \ldots A_{n}$ be a lattice oriented $O$-broken line. Let also $A_{0}=(1,0), A_{1}=\left(1, a_{0}\right), A_{n}=(p, q)$, where $\operatorname{gcd}(p, q)=1$, and $\left(a_{0}, a_{1}, \ldots, a_{2 n-2}\right)$ be the corresponding LSLS-sequence. Then the following holds:

$$
\left.\frac{q}{p}=\right] a_{0}, a_{1}, \ldots, a_{2 n-2}[\text {. }
$$

Proof. To prove this theorem we use an induction on the number of edges of the broken lines.

Base of induction. Suppose that a lattice oriented $O$-broken line has a unique edge, and the corresponding sequence is $\left(a_{0}\right)$. Then $A_{1}=\left(1, a_{0}\right)$ by the assumptions of the theorem. Therefore, we have $\left.\frac{a_{0}}{1}=\right] a_{0}[$.

Step of induction. Suppose that the statement of the theorem is correct for any lattice oriented $O$-broken line with $k$ edges. Let us prove the theorem for the arbitrary lattice oriented $O$-broken line with $k+1$ edges (and satisfying the conditions of the theorem).

Let $A_{0} \ldots A_{k+1}$ be a lattice oriented $O$-broken line with the following LSLSsequence $\left(a_{0}, a_{1}, \ldots, a_{2 k-1}, a_{2 k}\right)$. Let also

$$
A_{0}=(1,0), \quad A_{1}=\left(1, a_{0}\right), \quad \text { and } \quad A_{k+1}=(p, q) .
$$


Consider the lattice oriented $O$-broken line $B_{1} \ldots B_{k+1}$ with shorter LSLSsequence for it: $\left(a_{2}, a_{3}, \ldots, a_{2 k-2}, a_{2 k}\right)$. Let also

$$
B_{1}=(1,0), \quad B_{2}=\left(1, a_{2}\right), \quad \text { and } \quad B_{k+1}=\left(p^{\prime}, q^{\prime}\right) .
$$

By the induction assumption we have

$$
\left.\frac{q^{\prime}}{p^{\prime}}=\right] a_{2}, a_{3}, \ldots, a_{2 k}[\text {. }
$$

We extend the lattice oriented broken line $B_{1} \ldots B_{k+1}$ to the lattice oriented $O$-broken line $B_{0} B_{1} \ldots B_{k+1}$, where $B_{0}=\left(1+a_{0} a_{1},-a_{0}\right)$. Let the lattice LSLS-sequence for this broken line be $\left(b_{0}, b_{1}, \ldots, b_{2 k-1}, b_{2 k}\right)$. Note that

$$
\begin{aligned}
b_{0} & =\operatorname{sgn}\left(B_{0} O B_{1}\right) l \ell\left(B_{0} B_{1}\right)=\operatorname{sign}\left(a_{0}\right)\left|a_{0}\right|=a_{0}, \\
b_{1} & =\operatorname{sgn}\left(B_{0} O B_{1}\right) \operatorname{sgn}\left(B_{1} O B_{2}\right) \operatorname{sgn}\left(B_{0} B_{1} B_{2}\right) l \sin \angle B_{0} B_{1} B_{2} \\
& =\operatorname{sign} a_{0} \operatorname{sign} b_{2} \operatorname{sign}\left(a_{0} a_{1} b_{2}\right)\left|a_{1}\right|=a_{1}, \\
b_{l} & =a_{l}, \quad \text { for } \quad l=2, \ldots, 2 k .
\end{aligned}
$$

Consider a $\mathscr{L}_{+}$-linear transformation $\xi$ that takes the point $B_{0}$ to the point $(1,0)$, and $B_{1}$ to $\left(1, a_{0}\right)$. These two conditions uniquely define $\xi$ :

$$
\xi=\left(\begin{array}{cc}
1 & a_{1} \\
a_{0} & 1+a_{0} a_{1}
\end{array}\right)
$$

Since $B_{k+1}=\left(p^{\prime}, q^{\prime}\right)$, we have $\xi\left(B_{k+1}\right)=\left(p^{\prime}+a_{1} q^{\prime}, q^{\prime} a_{0}+p^{\prime}+p^{\prime} a_{0} a_{1}\right)$.

$$
\left.\frac{q^{\prime} a_{1}+p^{\prime}+p^{\prime} a_{0} a_{1}}{p^{\prime}+a_{1} q^{\prime}}=a_{0}+\frac{1}{a_{1}+q^{\prime} / p^{\prime}}=\right] a_{0}, a_{1}, a_{2}, a_{3}, \ldots, a_{2 n}[\text {. }
$$

Since, by Theorem 3.4 the lattice oriented broken lines $B_{0} B_{1} \ldots B_{k+1}$ and $A_{0} A_{1} \ldots A_{k+1}$ are $\mathscr{L}$-linear equivalent, $B_{0}=A_{0}$, and $B_{1}=A_{1}$, these broken lines coincide. Therefore, for the coordinates $(p, q)$ the following hold

$$
\left.\frac{q}{p}=\frac{q^{\prime} a_{0}+p^{\prime}+p^{\prime} a_{0} a_{1}}{p^{\prime}+a_{1} q^{\prime}}=\right] a_{0}, a_{1}, a_{2}, a_{3}, \ldots, a_{2 k}[\text {. }
$$

On Figure 6 we illustrate the step of induction with an example of lattice oriented $O$-broken line with the LSLS-sequence: $(1,-1,2,2,-1)$. We start (the left picture) with the broken line $B_{1} B_{2} B_{3}$ with the LSLS-sequence: $(2,2,-1)$. Note that the ratio of the coordinates of the point $B_{3}$ is $\left.-3 /-1=\right] 2: 2 ;-1[$. Then, (the picture in the middle) we extend the broken line $B_{1} B_{2} B_{3}$ to the broken line $B_{0} B_{1} B_{2} B_{3}$ with the LSLS-sequence: $(1,-1,2,2,-1)$. Finally 
(the right picture) we apply a corresponding $\mathscr{L}_{+}$-linear transformation $\xi$ to achieve the resulting broken line $A_{0} A_{1} A_{2} A_{3}$. Now the ratio of the coordinates of the point $A_{3}$ is $\left.-1 / 2=\right] 1:-1 ; 2 ; 2 ;-1[$.

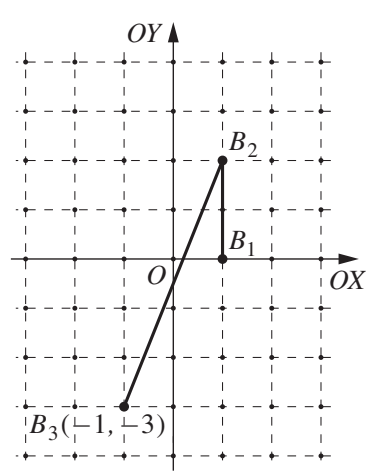

$-3 /-1=] 2: 2 ;-1[$

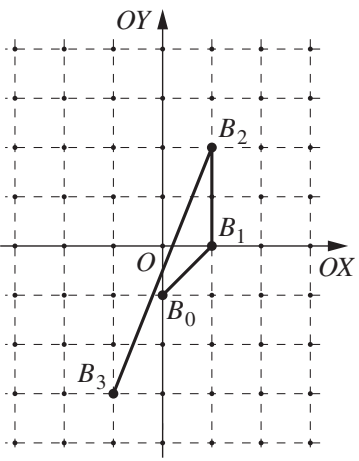

$B_{0}=\left(1+a_{0} a_{1},-a_{0}\right)$

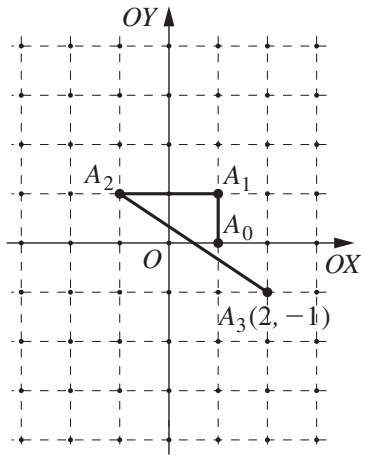

$-1 / 2=] 1:-1 ; 2 ; 2 ;-1[$

FIGURE 6 . The case of lattice oriented $O$-broken line with LSLS-sequence:

$(1,-1,2,2,-1)$.

We have proven the step of induction.

The proof of Theorem 3.5 is completed.

REMARK 3.6. Theorem 3.5 immediately implies the statement of Theorem 1.10. One should put the sail of an angle as an oriented-broken line $A_{0} A_{1} \ldots A_{n}$.

\subsection{Extended lattice angles. Sums for ordinary and extended lattice angles}

3.2.1. Equivalence classes of lattice oriented broken lines and the corresponding extended angles.

Definition 3.7. Consider a lattice point $V$. Two lattice oriented $V$-broken lines $l_{1}$ and $l_{2}$ are said to be equivalent if they have in common the first and the last vertices and the closed broken line generated by $l_{1}$ and the inverse of $l_{2}$ is homotopy equivalent to the point in $R^{2} \backslash\{V\}$.

An equivalence class of lattice oriented $V$-broken lines containing the broken line $A_{0} A_{1} \ldots A_{n}$ is called the extended lattice angle for the equivalence class of $A_{0} A_{1} \ldots A_{n}$ at the vertex $V$ (or, for short, extended angle) and denoted by $\angle\left(V, A_{0} A_{1} \ldots A_{n}\right)$.

We study the extended angles up to $\mathscr{L}_{+}$-congruence.

Definition 3.8. Two extended angles $\Phi_{1}$ and $\Phi_{2}$ are said to be $\mathscr{L}_{+-}$ congruent iff there exist a $\mathscr{L}_{+}$-affine transformation sending the class of lattice 
oriented broken lines corresponding to $\Phi_{1}$ to the class of lattice oriented broken lines corresponding to $\Phi_{2}$. We denote this by $\Phi_{1} \hat{\cong} \Phi_{2}$.

3.2.2. Revolution numbers for extended angles. Let $r=\{V+\lambda \bar{v} \mid \lambda \geq 0\}$ be the oriented ray for an arbitrary vector $\bar{v}$ with the vertex at $V$, and $A B$ be an oriented (from $A$ to $B$ ) segment not contained in the ray $r$. Suppose also, that the vertex $V$ of the ray $r$ is not contained in the segment $A B$. We denote by $\#(r, V, A B)$ the following number:

$$
\#(r, V, A B)= \begin{cases}0, & A B \cap r=\emptyset \\ \frac{1}{2} \operatorname{sgn}(A(A-\bar{v}) B), & A B \cap r \in\{A, B\} \\ \operatorname{sgn}(A(A-\bar{v}) B), & A B \cap r \in A B \backslash\{A, B\},\end{cases}
$$

and call it the intersection number of the ray $r$ and the segment $A B$.

Definition 3.9. Let $A_{0} A_{1} \ldots A_{n}$ be some lattice oriented broken line, and let $r$ be an oriented ray $\{V+\lambda \bar{v} \mid \lambda \geq 0\}$. Suppose that the ray $r$ does not contain the edges of the broken line, and the broken line does not contain the point $V$. We call the number

$$
\sum_{i=1}^{n} \#\left(r, V, A_{i-1} A_{i}\right)
$$

the intersection number of the ray $r$ and the lattice oriented broken line $A_{0} A_{1} \ldots A_{n}$, and denote it by $\#\left(r, V, A_{0} A_{1} \ldots A_{n}\right)$.

Definition 3.10. Consider an arbitrary extended angle $\angle\left(V, A_{0} A_{1} \ldots A_{n}\right)$. Denote the rays $\left\{V+\lambda \overline{V A_{0}} \mid \lambda \geq 0\right\}$ and $\left\{V-\lambda \overline{V A_{0}} \mid \lambda \geq 0\right\}$ by $r_{+}$and $r_{-}$ respectively. The number

$$
\frac{1}{2}\left(\#\left(r_{+}, V, A_{0} A_{1} \ldots A_{n}\right)+\#\left(r_{-}, V, A_{0} A_{1} \ldots A_{n}\right)\right)
$$

is called the lattice revolution number for the extended angle $\angle\left(V, A_{0} A_{1} \ldots A_{n}\right)$, and denoted by $\#\left(\angle\left(V, A_{0} A_{1} \ldots A_{n}\right)\right)$. We say also that $\#\left(\angle\left(V, A_{0}\right)\right)=0$.

Let us give some examples. Let $O=(0,0), A=(1,0), B=(0,1)$, $C=(-1,-1)$, then

$$
\begin{gathered}
\#(\angle(O, A))=0, \quad \#(\angle(O, A B))=\frac{1}{4}, \\
\#(\angle(O, A B C A))=1, \quad \#(\angle(O, A C B))=-\frac{3}{4} .
\end{gathered}
$$

Now we show that the definition of revolution number is correct. 
PROPOSITION 3.11. The revolution number of any extended angle is welldefined.

Proof. Consider an arbitrary extended angle $\angle\left(V, A_{0} A_{1} \ldots A_{n}\right)$. Let

$$
r_{+}=\left\{V+\lambda \overline{V A_{0}} \mid \lambda \geq 0\right\} \quad \text { and } \quad r_{-}=\left\{V-\lambda \overline{V A_{0}} \mid \lambda \geq 0\right\} .
$$

Since the lattice oriented broken line $A_{0} A_{1} \ldots A_{n}$ is at unit distance from the point $V$, any segment of this broken line is at unit distance from $V$. Thus, the broken line does not contain $V$, and the rays $r_{+}$and $r_{-}$do not contain edges of the curve.

Suppose that

$$
\angle\left(V, A_{0} A_{1} \ldots A_{n}\right)=\angle\left(V^{\prime}, A_{0}^{\prime} A_{1}^{\prime} \ldots A_{m}^{\prime}\right) .
$$

This implies that $V=V^{\prime}, A_{0}=A_{0}^{\prime}, A_{n}=A_{m}^{\prime}$, and the broken line $A_{0} A_{1} \ldots A_{n} A_{m-1}^{\prime} \ldots A_{1}^{\prime} A_{0}^{\prime}$ is homotopy equivalent to the point in $\mathrm{R}^{2} \backslash\{V\}$. Thus,

$$
\begin{aligned}
\#\left(L\left(V, A_{0} A_{1} \ldots A_{n}\right)\right)-\#\left(L\left(V, A_{0}^{\prime} A_{1}^{\prime} \ldots A_{m}^{\prime}\right)\right) \\
=\frac{1}{2}\left(\#\left(r_{+}, V, A_{0} A_{1} \ldots A_{n} A_{m-1}^{\prime} \ldots A_{1}^{\prime} A_{0}^{\prime}\right)\right. \\
\left.\quad+\#\left(r_{-}, V, A_{0} A_{1} \ldots A_{n} A_{m-1}^{\prime} \ldots A_{1}^{\prime} A_{0}^{\prime}\right)\right) \\
=0+0=0 .
\end{aligned}
$$

Hence,

$$
\#\left(\angle\left(V, A_{0} A_{1} \ldots A_{n}\right)\right)=\#\left(\angle\left(V^{\prime}, A_{0}^{\prime} A_{1} \ldots A_{m}^{\prime}\right)\right) .
$$

Therefore, the revolution number of any extended angle is well-defined.

PROPOSITION 3.12. The revolution number of extended angles is invariant under the group action of the $\mathscr{L}_{+}$-affine transformations.

3.2.3. Zero ordinary angles. For the next theorem we will need to define zero ordinary angles and their trigonometric functions. Let $A, B$, and $C$ be three lattice points of the same lattice straight line. Suppose that $B$ is distinct to $A$ and $C$ and the rays $B A$ and $B C$ coincide. We say that the ordinary angle with the vertex at $B$ and the rays $B A$ and $B C$ is zero. Suppose $\angle A B C$ is zero, put by definition

$$
\operatorname{lsin}(\angle A B C)=0, \quad \operatorname{lcos}(\angle A B C)=1, \quad \operatorname{ltan}(\angle A B C)=0 .
$$

Denote by $\operatorname{larctan}(0)$ the angle $\angle A O A$ where $A=(1,0)$, and $O$ is the origin. 
3.2.4. On normal forms of extended angles. Let us formulate and prove a theorem on normal forms of extended angles. We use the following notation: by the sequence

$$
\left(\left(a_{0}, \ldots, a_{n}\right) \times k \text {-times }, b_{0}, \ldots, b_{m}\right),
$$

where $k \geq 0$, we denote the following sequence:

$$
(\underbrace{a_{0}, \ldots, a_{n}, a_{0}, \ldots, a_{n}, \ldots, a_{0}, \ldots, a_{n}}_{k \text {-times }}, b_{0}, \ldots, b_{m}) .
$$

Definition 3.13. I) Suppose $O$ be the origin, $A_{0}$ be the point $(1,0)$. We say that the extended angle $\angle\left(O, A_{0}\right)$ is of the type I and denote it by $0 \pi+\operatorname{larctan}(0)$ (or 0 , for short). The empty sequence is said to be characteristic for the angle $0 \pi+\operatorname{larctan}(0)$.

Consider a lattice oriented $O$-broken line $A_{0} A_{1} \ldots A_{s}$, where $O$ is the origin. Let also $A_{0}$ be the point $(1,0)$, and the point $A_{1}$ be on the straight line $x=1$. If the LSLS-sequence of the extended angle $\Phi_{0}=\angle\left(O, A_{0} A_{1} \ldots A_{s}\right)$ coincides with the following sequence (we call it characteristic sequence for the corresponding angle):

$\left.\mathrm{II}_{k}\right)((1,-2,1,-2) \times(k-1)$-times, $1,-2,1)$, where $k \geq 1$, then we denote the angle $\Phi_{0}$ by $k \pi+\operatorname{larctan}(0)$ (or $k \pi$, for short) and say that $\Phi_{0}$ is of the type $\mathrm{II}_{k}$;

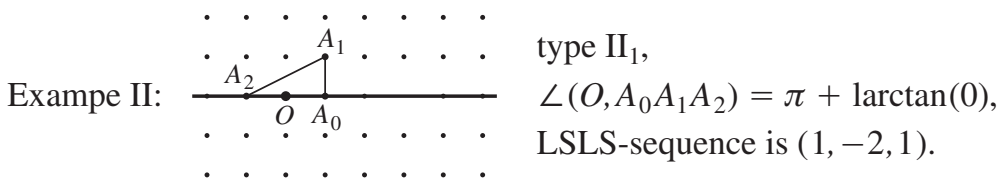

$\left.\mathrm{III}_{k}\right)((-1,2,-1,2) \times(k-1)$-times, $-1,2,-1)$, where $k \geq 1$, then we denote the angle $\Phi_{0}$ by $-k \pi+\operatorname{larctan}(0)$ (or $-k \pi$, for short) and say that $\Phi_{0}$ is of the type $\mathrm{III}_{k}$; 


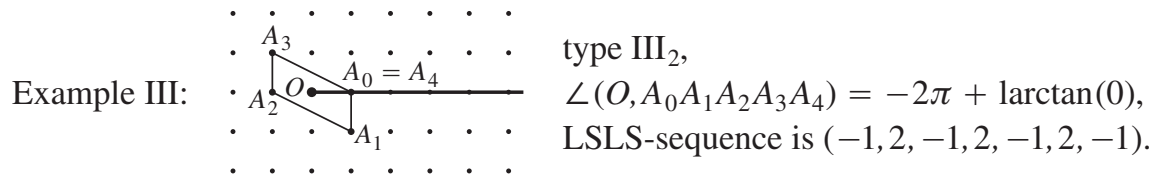

$\left.\mathrm{IV}_{k}\right)\left((1,-2,1,-2) \times k\right.$-times, $\left.a_{0}, \ldots, a_{2 n}\right)$, where $k \geq 0, n \geq 0, a_{i}>0$, for $i=0, \ldots, 2 n$, then we denote the angle $\Phi_{0}$ by $k \pi+\operatorname{larctan}\left(\left[a_{0}, a_{1}, \ldots\right.\right.$, $\left.a_{2 n}\right]$ ) and say that $\Phi_{0}$ is of the type $\mathrm{IV}_{k}$;

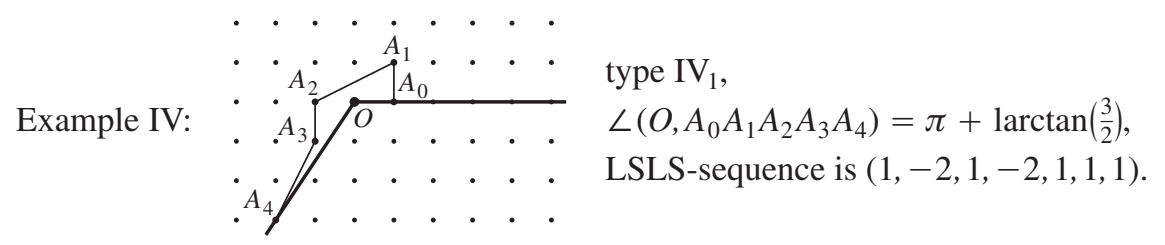

$\left.\mathrm{V}_{k}\right)\left((-1,2,-1,2) \times k\right.$-times, $\left.a_{0}, \ldots, a_{2 n}\right)$, where $k>0, n \geq 0, a_{i}>0$, for $i=0, \ldots, 2 n$, then we denote the angle $\Phi_{0}$ by $-k \pi+\operatorname{larctan}\left(\left[a_{0}, a_{1}, \ldots\right.\right.$, $\left.a_{2 n}\right]$ ) and say that $\Phi_{0}$ is of the type $\mathrm{V}_{k}$.

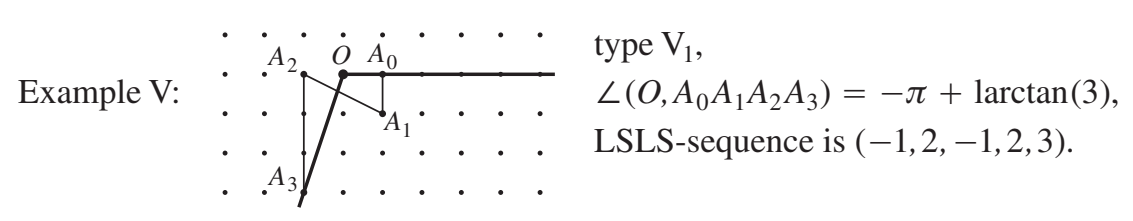

THEOREM 3.14. For any extended angle $\Phi$ there exist a unique type among the types $\mathrm{I}-\mathrm{V}$ and a unique extended angle $\Phi_{0}$ of that type such that $\Phi_{0}$ is $\mathscr{L}_{+}$-congruent to $\Phi$.

The extended angle $\Phi_{0}$ is said to be the normal form for the extended angle $\Phi$.

For the proof of Theorem 3.14 we need the following lemma.

Lemma 3.15. Let $m, k \geq 1$, and $a_{i}>0$ for $i=0, \ldots, 2 n$ be some integers.

a) Suppose the LSLS-sequences for the extended angles $\Phi_{1}$ and $\Phi_{2}$ are respectively

$$
\left((1,-2,1,-2) \times(k-1) \text {-times }, 1,-2,1,-2, a_{0}, \ldots, a_{2 n}\right)
$$

and

$$
\left((1,-2,1,-2) \times(k-1) \text {-times }, 1,-2,1, m, a_{0}, \ldots, a_{2 n}\right),
$$

then $\Phi_{1}$ is $\mathscr{L}_{+}$-congruent to $\Phi_{2}$. 
b) Suppose the LSLS-sequences for the extended angles $\Phi_{1}$ and $\Phi_{2}$ are respectively

$$
\left((-1,2,-1,2) \times(k-1) \text {-times },-1,2,-1, m, a_{0}, \ldots, a_{2 n}\right)
$$

and

$$
\left((-1,2,-1,2) \times(k-1) \text {-times },-1,2,-1,2, a_{0}, \ldots, a_{2 n}\right),
$$

then $\Phi_{1}$ is $\mathscr{L}_{+}$-congruent to $\Phi_{2}$.

Proof. We prove the first statement of the lemma. Suppose that $m$ is integer, $k$ is positive integer, and $a_{i}$ for $i=0, \ldots, 2 n$ are positive integers.

Let us construct the angle $\Psi_{1}$ with vertex at the origin for the lattice oriented broken line $A_{0} \ldots A_{2 k+n+1}$, corresponding to the LSLS-sequence

$$
\left((1,-2,1,-2) \times(k-1) \text {-times, } 1,-2,1,-2, a_{0}, \ldots, a_{2 n}\right),
$$

such that $A_{0}=(1,0), A_{1}=(1,1)$. Note that

$$
\left\{\begin{array}{rlrl}
A_{2 l} & =\left((-1)^{l}, 0\right), & & \text { for } \quad l<k-1 \\
A_{2 l+1} & =\left((-1)^{l},(-1)^{l}\right), & & \text { for } \quad l<k-1 \\
A_{2 k} & =\left((-1)^{k}, 0\right) & & \\
A_{2 k+1} & =\left((-1)^{k},(-1)^{k} a_{0}\right) & &
\end{array} .\right.
$$

Let us construct the angle $\Psi_{2}$ with vertex at the origin for the lattice oriented broken line $B_{0} \ldots B_{2 k+n+1}$, corresponding to the LSLS-sequence

$$
\left((1,-2,1,-2) \times(k-1) \text {-times, } 1,-2,1, m, a_{0}, \ldots, a_{2 n}\right) .
$$

such that $B_{0}=(1,0), B_{1}=(-m-1,1)$. Note also that

$$
\left\{\begin{array}{rlrl}
B_{2 l} & =\left((-1)^{l}, 0\right), & & \text { for } \quad l<k-1 \\
B_{2 l+1} & =\left((-1)^{l}(-m-1),(-1)^{l}\right), & & \text { for } \quad l<k-1 \\
B_{2 k} & =\left((-1)^{k}, 0\right) & & \\
B_{2 k+1} & =\left((-1)^{k},(-1)^{k} a_{0}\right) & &
\end{array} .\right.
$$

From the above we know, that the points $A_{2 k}$ and $A_{2 k+1}$ coincide with the points $B_{2 k}$ and $B_{2 k+1}$ respectively. Since the remaining parts of both LSLSsequences (i. e. $\left.\left(a_{0}, \ldots, a_{2 n}\right)\right)$ coincide, the point $A_{l}$ coincide with the point $B_{l}$ for $l>2 k$. 
Since the lattice oriented broken lines $A_{0} \ldots A_{2 k}$ and $B_{0} \ldots B_{2 k}$ are of the same equivalence class, and the point $A_{l}$ coincide with the point $B_{l}$ for $l>2 k$, we obtain

$$
\Psi_{1}=\angle\left(O, A_{0} \ldots A_{2 k+n+1}\right)=\angle\left(O, B_{0} \ldots B_{2 k+n+1}\right)=\Psi_{2} .
$$

Therefore, by Theorem 3.4 we have the following:

$$
\Phi_{1} \hat{\cong} \Psi_{1}=\Psi_{2} \hat{\cong} \Phi_{2} .
$$

This concludes the proof of Lemma 3.15a.

Since the proof of Lemma 3.15b almost completely repeats the proof of Lemma 3.15a, we omit the proof of Lemma 3.15b here.

Proof of Theorem 3.14. First, we prove that any two distinct extended angles listed in Definition 3.13 are not $\mathscr{L}_{+}$-congruent. Let us note that the revolution numbers of extended angles distinguish the types of the angles. The revolution number for the extended angle of the type $\mathrm{I}$ is 0 . The revolution number for the extended angle of the type $\mathrm{II}_{k}$ is $1 / 2(k+1)$ where $k \geq 0$. The revolution number for the extended angle of the type $\mathrm{III}_{k}$ is $-1 / 2(k+1)$ where $k \geq 0$. The revolution number for the extended angles of the type $\mathrm{IV}_{k}$ is $1 / 4+1 / 2 k$ where $k \geq 0$. The revolution number for the extended angles of the type $\mathrm{V}_{k}$ is $1 / 4-1 / 2 k$ where $k>0$.

So we have proven that two extended angles of different types are not $\mathscr{L}_{+}^{-}$ congruent. For the types $\mathrm{I} \mathrm{II}_{k}$, and $\mathrm{III}_{k}$ the proof is completed, since any such type consists of the unique extended angle.

Let us prove that normal forms of the same type $\mathrm{IV}_{k}$ (or of the same type $\mathrm{V}_{k}$ ) are not $\mathscr{L}_{+}$-congruent for any integer $k \geq 0$ (or $k>0$ ). Consider an extended angle $\Phi=k \pi+\operatorname{larctan}\left(\left[a_{0}, a_{1}, \ldots, a_{2 n}\right]\right)$. Suppose that a lattice oriented $O$-broken line $A_{0} A_{1} \ldots A_{m}$, where $m=2|k|+n+1$ defines the angle $\Phi$. Let also that the LSLS-sequence for this broken line be characteristic.

Suppose, that $k$ is even, then the ordinary angle $\angle A_{0} O A_{m}$ is $\mathscr{L}_{+}$-congruent to the ordinary angle $\operatorname{larctan}\left(\left[a_{0}, a_{1}, \ldots, a_{2 n}\right]\right)$. This angle is a $\mathscr{L}_{+}$-affine invariant for the extended angle $\Phi$. This invariant distinguish the extended angles of type $\mathrm{IV}_{k}$ (or $\mathrm{V}_{k}$ ) with even $k$.

Suppose, that $k$ is odd, then denote $B=O+\overline{A_{0} O}$. The ordinary angle $\angle B V A_{m}$ is $\mathscr{L}_{+}$-congruent to the ordinary angle $\operatorname{larctan}\left(\left[a_{0}, a_{1}, \ldots, a_{2 n}\right]\right)$. This angle is a $\mathscr{L}_{+}$-affine invariant for the extended angle $\Phi$. This invariant distinguish the extended angles of type $\mathrm{IV}_{k}$ (or $\mathrm{V}_{k}$ ) with odd $k$.

Therefore, the extended angles listed in Definition 3.13 are not $\mathscr{L}_{+}$-congruent.

Now we prove that an arbitrary extended angle is $\mathscr{L}_{+}$-congruent to one of the extended angles of the types I-V. 
Consider an arbitrary extended angle $\angle\left(V, A_{0} A_{1} \ldots A_{n}\right)$ and denote it by $\Phi$. If $\#(\Phi)=k / 2$ for some integer $k$, then $\Phi$ is $\mathscr{L}_{+}$-congruent to an angle of one of the types I-III. Let \# $(\Phi)=1 / 4$, then the extended angle $\Phi$ is $\mathscr{L}_{+}$-congruent to the extended angle defined by the sail of the ordinary angle $\angle A_{0} V A_{n}$ of the type $\mathrm{IV}_{0}$.

Suppose now, that $\#(\Phi)=1 / 4+k / 2$ for some positive integer $k$, then one of its LSLS-sequence is of the following form:

$$
\left((1,-2,1,-2) \times(k-1) \text {-times, } 1,-2,1, m, a_{0}, \ldots, a_{2 n}\right),
$$

where $a_{i}>0$, for $i=0, \ldots, 2 n$. By Lemma 3.15 the extended angle defined by this sequence is $\mathscr{L}_{+}$-congruent to an extended angle of the type $\mathrm{IV}_{k}$ defined by the sequence

$$
\left((1,-2,1,-2) \times(k-1) \text {-times, } 1,-2,1,-2, a_{0}, \ldots, a_{2 n}\right) .
$$

Finally, let $\#(\Phi)=1 / 4-k / 2$ for some positive integer $k$, then one of its LSLS-sequence is of the following form:

$$
\left((-1,2,-1,2) \times(k-1) \text {-times, }-1,2,-1, m, a_{0}, \ldots, a_{2 n}\right),
$$

where $a_{i}>0$, for $i=0, \ldots, 2 n$. By Lemma 3.15 the extended angle defined by this sequence is $\mathscr{L}_{+}$-congruent to an extended angle of the type $\mathrm{V}_{k}$ defined by the sequence

$$
\left((-1,2,-1,2) \times(k-1) \text {-times, }-1,2,-1,2, a_{0}, \ldots, a_{2 n}\right) .
$$

This completes the proof of Theorem 3.14.

Let us finally give the definition of trigonometric functions for the extended angles and describe some relations between ordinary and extended angles.

Definition 3.16. Consider an arbitrary extended angle $\Phi$ with the normal form $k \pi+\varphi$ for some ordinary (possible zero) angle $\varphi$ and for an integer $k$.

a) The ordinary angle $\varphi$ is said to be associated with the extended angle $\Phi$.

b) The numbers $1 \tan (\varphi), \operatorname{lin}(\varphi)$, and $\operatorname{los}(\varphi)$ are called the lattice tangent, the lattice sine, and the lattice cosine of the extended angle $\Phi$.

Since all sails for ordinary angles are lattice oriented broken lines, the set of all ordinary angles is naturally embedded into the set of extended angles.

Definition 3.17. For an ordinary angle $\varphi$ the angle

$$
0 \pi+\operatorname{larctan}(\operatorname{ltan} \varphi)
$$

is said to be corresponding to the angle $\varphi$ and denoted by $\bar{\varphi}$. 
From Theorem 3.14 it follows that for every ordinary angle $\varphi$ there exists and unique an extended angle $\bar{\varphi}$ corresponding to $\varphi$. Therefore, two ordinary angles $\varphi_{1}$ and $\varphi_{2}$ are $\mathscr{L}$-congruent iff the corresponding lattice angles $\bar{\varphi}_{1}$ and $\bar{\varphi}_{2}$ are $\mathscr{L}_{+}$-congruent.

3.2.5. Opposite extended angles. Sums of extended angles. Sums of ordinary angles. Consider an extended angle $\Phi$ with the vertex $V$ for some equivalence class of a given lattice oriented broken line. The extended angle $\Psi$ with the vertex $V$ for the equivalence class of the inverse lattice oriented broken line is called opposite to the given one and denoted by $-\Phi$.

Proposition 3.18. For any extended angle $\Phi \stackrel{\cong}{\cong} \pi+\varphi$ we have:

$$
-\Phi \cong(-k-1) \pi+(\pi-\varphi)
$$

Let us introduce the definition of sums of ordinary and extended angles.

Definition 3.19. Consider arbitrary extended angles $\Phi_{i}, i=1, \ldots, l$. Let the characteristic sequences for the normal forms of $\Phi_{i}$ be $\left(a_{0, i}, a_{1, i}, \ldots, a_{2 n_{i}, i}\right)$ for $i=1, \ldots, l$. Let $M=\left(m_{1}, \ldots, m_{l-1}\right)$ be some $(l-1)$-tuple of integers. The normal form of any extended angle, corresponding to the following LSLSsequence

$$
\left(a_{0,1}, a_{1,1}, \ldots, a_{2 n_{1}, 1}, m_{1}, a_{0,2}, \ldots, a_{2 n_{2}, 2}, m_{2}, \ldots, m_{l-1}, a_{0, l}, \ldots, a_{2 n_{l}, l}\right),
$$

is called the $M$-sum of extended angles $\Phi_{i}(i=1, \ldots, l)$ and denoted by

$$
\sum_{M, i=1}^{l} \Phi_{i}, \quad \text { or equivalently by } \Phi_{1}+{ }_{m_{1}} \Phi_{2}+{ }_{m_{2}} \cdots+{ }_{m_{l-1}} \Phi_{l} \text {. }
$$

Proposition 3.20. The $M$-sum of extended angles $\Phi_{i}(i=1, \ldots, l)$ is well-defined.

Let us say a few words about properties of $M$-sums.

Notice that $M$-sum of extended angles is non-associative. For example, let $\Phi_{1} \hat{\cong} \operatorname{larctan} 2, \Phi_{2} \cong \operatorname{\cong arctan}(3 / 2)$, and $\Phi_{3} \cong \operatorname{\cong } \arctan 5$. Then

$$
\begin{aligned}
\Phi_{1}+_{-1} \Phi_{2}+_{-1} \Phi_{3} & =\pi+\operatorname{larctan}(4), \\
\Phi_{1}+_{-1}\left(\Phi_{2}+_{-1} \Phi_{3}\right) & =2 \pi \\
\left(\Phi_{1}+_{-1} \Phi_{2}\right)+_{-1} \Phi_{3} & =\operatorname{larctan}(1)
\end{aligned}
$$


The $M$-sum of extended angles is non-commutative. For example, let $\Phi_{1} \cong$ $\operatorname{larctan} 1$, and $\Phi_{2} \hat{\cong} \operatorname{larctan} 5 / 2$. Then

$$
\Phi_{1}+{ }_{1} \Phi_{2}=\operatorname{larctan}(12 / 7) \neq \operatorname{larctan}(13 / 5)=\Phi_{2}+{ }_{1} \Phi_{1} .
$$

Remark 3.21. The $M$-sum of extended angles is naturally extended to the sum of classes of $\mathscr{L}_{+}$-congruences of extended angles.

We conclude this section with the definition of sums of ordinary angles.

Definition 3.22. Consider ordinary angles $\alpha_{i}$, where $i=1, \ldots, l$. Let $\bar{\alpha}_{i}$ be the corresponding extended angles for $\alpha_{i}$, and $M=\left(m_{1}, \ldots, m_{l-1}\right)$ be some $(l-1)$-tuple of integers. The ordinary angle $\varphi$ associated with the extended angle

$$
\Phi=\bar{\alpha}_{1}+_{m_{1}} \bar{\alpha}_{2}+_{m_{2}} \cdots+{ }_{m_{l-1}} \bar{\alpha}_{l} .
$$

is called the $M$-sum of ordinary angles $\alpha_{i}(i=1, \ldots, l)$ and denoted by

$$
\sum_{M, i=1}^{l} \alpha_{i}, \quad \text { or equivalently by } \alpha_{1}+{ }_{m_{1}} \alpha_{2}+_{m_{2}} \cdots+_{m_{l-1}} \alpha_{l} .
$$

REMARK 3.23. Note that the sum of ordinary angles is naturally extended to the classes of $\mathscr{L}$-congruences of lattice angles.

\section{Relations between extended and ordinary lattice angles. Proof of the first statement of Theorem 2.2}

Throughout this section we again fix some lattice basis and use the system of coordinates $O X Y$ corresponding to this basis.

\subsection{On relations between continued fractions for lattice oriented broken lines and the lattice tangents of the corresponding extended angles}

For a real number $r$ we denote by $\lfloor r\rfloor$ the maximal integer not greater than $r$.

Theorem 4.1. Consider an extended angle $\Phi=\angle\left(V, A_{0} A_{1} \ldots A_{n}\right)$. Suppose, that the normal form for $\Phi$ is $k \pi+\varphi$ for some integer $k$ and an ordinary angle $\varphi$. Let $\left(a_{0}, a_{1}, \ldots, a_{2 n-2}\right)$ be the LSLS-sequence for the lattice oriented broken line $A_{0} A_{1} \ldots A_{n}$. Suppose that

$$
] a_{0}, a_{1}, \ldots, a_{2 n-2}[=q / p .
$$


Then the following holds:

$$
\varphi \cong\left\{\begin{array}{ll}
\operatorname{larctan}(1), & \text { if } q / p=\infty \\
\operatorname{larctan}(q / p), & \text { if } q / p \geq 1 \\
\operatorname{larctan}\left(\frac{|q|}{|p|-\lfloor(|p|-1) /|q|\rfloor|q|}\right), & \text { if } 0<q / p<1 \\
0, & \text { if } q / p=0 \\
\pi-\operatorname{larctan}\left(\frac{|q|}{|p|-\lfloor(|p|-1) /|q|\rfloor|q|}\right), & \text { if }-1<q / p<0 \\
\pi-\operatorname{larctan}(-q / p), & \text { if } q / p \leq-1
\end{array} .\right.
$$

Proof. Consider the following linear coordinates $(*, *)^{\prime}$ on the plane, associated with the lattice oriented $V$-broken line $A_{0} A_{1} \ldots A_{n}$. Let the origin $O^{\prime}$ be at the vertex $V,(1,0)^{\prime}=A_{0}$, and $(1,1)^{\prime}=A_{0}+\frac{1}{a_{0}} \operatorname{sgn}\left(A_{0} O^{\prime} A_{1}\right) \overline{A_{0} A_{1}}$. The other coordinates are uniquely defined by linearity. We denote this system of coordinates by $O^{\prime} X^{\prime} Y^{\prime}$.

The set of integer points for the coordinate system $O^{\prime} X^{\prime} Y^{\prime}$ coincides with the set of lattice points of the plane. The basis of vectors $(1,0)^{\prime}$ and $(0,1)^{\prime}$ defines a positive orientation.

Suppose that the new coordinates of the point $A_{n}$ are $\left(p^{\prime}, q^{\prime}\right)^{\prime}$. Then by Theorem 3.5 we have $q^{\prime} / p^{\prime}=q / p$. This directly implies the statement of the theorem for the cases $q^{\prime}>p^{\prime}>0, q^{\prime} / p^{\prime}=0$, and $q^{\prime} / p^{\prime}=\infty$.

Suppose now that $p^{\prime}>q^{\prime}>0$. Consider the ordinary angle $\varphi=\angle A_{0} P A_{n}$. Let $B_{0} \ldots B_{m}$ be the sail for it. The direct calculations show that the point

$$
D=B_{0}+\frac{\overline{B_{0} B_{1}}}{1 \ell\left(B_{0} B_{1}\right)}
$$

coincides with the point $\left(1+\left\lfloor\left(p^{\prime}-1\right) / q^{\prime}\right\rfloor, 1\right)$ in the system of coordinates $O^{\prime} X^{\prime} Y^{\prime}$.

Consider the $\mathscr{L}_{+}$-linear (in the coordinates $O^{\prime} X^{\prime} Y^{\prime}$ ) transformation $\xi$ that takes the point $A_{0}=B_{0}$ to itself, and the point $D$ to $(1,1)^{\prime}$. These conditions uniquely identify $\xi$.

$$
\xi=\left(\begin{array}{cc}
1 & -\left\lfloor\left(p^{\prime}-1\right) / q^{\prime}\right\rfloor \\
0 & 1
\end{array}\right)
$$

The transformation $\xi$ takes the point $A_{n}=B_{m}$ with the coordinates $\left(p^{\prime}, q^{\prime}\right)$ to the point with the coordinates $\left(p^{\prime}-\left\lfloor\left(p^{\prime}-1\right) / q^{\prime}\right\rfloor q^{\prime}, q^{\prime}\right)^{\prime}$. Since $q^{\prime} / p^{\prime}=q / p$, we obtain the following

$$
\varphi=\operatorname{larctan}\left(\frac{q^{\prime}}{p^{\prime}-\left\lfloor\left(p^{\prime}-1\right) / q^{\prime}\right\rfloor q^{\prime}}\right)=\operatorname{larctan}\left(\frac{q}{p-\lfloor(p-1) / q\rfloor q}\right) .
$$


The proof for the case $q^{\prime}>0$ and $p^{\prime}<0$ repeats the described cases after taking to the consideration the adjacent angles.

Finally, the case of $q^{\prime}<0$ repeats all previous cases by the central symmetry (centered at the point $O^{\prime}$ ) reasons.

This completes the proof of Theorem 4.1.

COROLlary 4.2. The revolution number and the continued fraction for a lattice oriented broken line at unit distance from the vertex uniquely define the $\mathscr{L}_{+}$-congruence class of the corresponding extended angle.

\subsection{Proof of Theorem 2.2a: two preliminary lemmas}

We say that the lattice point $P$ is at lattice distance $k$ from the lattice segment $A B$ if the lattice vectors of the segment $A B$ and the vector $\overline{A P}$ generate a sublattice of the lattice of index $k$.

Definition 4.3. Consider a lattice triangle $\triangle A B C$. Denote the number of lattice points at unit lattice distance from the segment $A B$ and contained in the (closed) triangle $\triangle A B C$ by $\ell_{1}(A B ; C)$ (see on Figure 7).

Note that all lattice points at lattice unit lattice distance from the segment $A B$ in the (closed) lattice triangle $\triangle A B C$ are contained in one straight line parallel to the straight line $A B$. Besides, the integer $1 \ell_{1}(A B ; C)$ is positive for any triangle $\triangle A B C$.

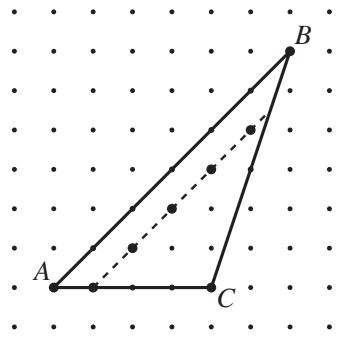

Figure 7. For the given triangle $\triangle A B C$ we have $\ell_{1}(A B ; C)=5$.

Now we prove the following lemma.

Lemma 4.4. For any lattice triangle $\triangle A B C$ the following holds

$$
\overline{\angle C A B}+1 \ell(A B)-1 \ell_{1}(A B ; C)-1, \overline{L A B C}+1 \ell(B C)-1 \ell_{1}(B C ; A)-1 \overline{\angle B C A}=\pi .
$$

PRoof. Consider an arbitrary lattice triangle $\triangle A B C$. Suppose that the pair of vectors $\overline{B A}$ and $\overline{B C}$ defines the positive orientation of the plane (otherwise we apply to the triangle $\triangle A B C$ some $\mathscr{L}$-affine transformation changing the 
orientation and come to the same position). Denote (see Figure 9 below): $D=A+\overline{B C}$, and $E=A+\overline{A C}$.

Since $C A D B$ is a parallelogram, the triangle $\triangle B A D$ is $\mathscr{L}_{+}$-congruent to the triangle $\triangle A B C$. Thus, the angle $\angle B A D$ is $\mathscr{L}_{+}$-congruent to the angle $\angle A B C$, and $1 \ell_{1}(B A ; D)=1 \ell_{1}(A B ; C)$. Since $E A B D$ is a parallelogram, the triangle $\triangle A E D$ is $\mathscr{L}_{+}$-congruent to the triangle $\triangle B A D$, and hence is $\mathscr{L}_{+}$-congruent to the triangle $\triangle A B C$. Thus, Thus, $\angle D A E$ is $\mathscr{L}_{+}$-congruent to $\angle B C A$, and $1 \ell_{1}(D A ; E)=1 \ell_{1}(B C ; A)$.

Let $A_{0} \ldots A_{n}$ be the sail of $\angle C A B$ with the corresponding LLS-sequence $\left(a_{0}, \ldots, a_{2 n-2}\right)$. Let $B_{0} B_{1} \ldots B_{m}$ be the sail of $\angle B A D$ (where $B_{0}=A_{n}$ ) with the corresponding LLS-sequence $\left(b_{0}, \ldots, b_{2 m-2}\right)$. And let $C_{0} C_{1} \ldots C_{l}$ be the sail of $\angle D A E$ (where $C_{0}=B_{m}$ ) with the corresponding LLS-sequence $\left(c_{0}, \ldots, c_{2 l-2}\right)$.

Consider now the lattice oriented broken line

$$
A_{0} \ldots A_{n} B_{1} B_{2} \ldots B_{m} C_{1} C_{2} \ldots C_{l} .
$$

The LSLS-sequence for this broken line is

$$
\left(a_{0}, \ldots, a_{2 n-2}, t, b_{0}, \ldots, b_{2 m-2}, u, c_{0}, \ldots, c_{2 l-2}\right),
$$

where integers $t$ and $u$ are integers defined by the broken line. By definition of the sum of extended angles this sequence defines the extended angle

$$
\overline{\angle C A B}+{ }_{t} \overline{\angle B A D}+{ }_{u} \overline{\angle D A E} .
$$

By Theorem 4.1, we have $\overline{\angle C A B}+{ }_{t} \overline{\angle B A D}+{ }_{u} \overline{\angle D A E}=\pi$.

We compute now the integer $t$. Denote by $A_{n}^{\prime}$ the closest lattice point to the point $A_{n}$ and distinct to $A_{n}$ in the segment $A_{n-1} A_{n}$. Consider the set of lattice points at unit lattice distance from the segment $A B$ and lying in the halfplane with the boundary straight line $A B$ and containing the point $D$. This set coincides with the following set (See Figure 8):

$$
\left\{A_{n, k}=A_{n}+\overline{A_{n}^{\prime} A_{n}}+k \overline{A A_{n}} \mid k \in \mathrm{Z}\right\} .
$$

Since $A_{n,-2}=A+\overline{A_{n}^{\prime} A}$, the points $A_{n, k}$ for $k \leq-2$ are in the closed half-plane bounded by the straight line $A C$ and not containing the point $B$.

Since $A_{n,-1}=A+\overline{A_{n}^{\prime} A_{n}}$, the points $A_{n, k}$ for $k \geq-1$ are in the open half-plane bounded by the straight line $A C$ and containing the point $B$.

The intersection of the parallelogram $A E D B$ and the open half-plane bounded by the straight line $A C$ and containing the point $B$ contains exactly $1 \ell(A B)$ points of the described set: only the points $A_{n, k}$ with $-1 \leq k \leq 1 \ell(A B)-2$. 


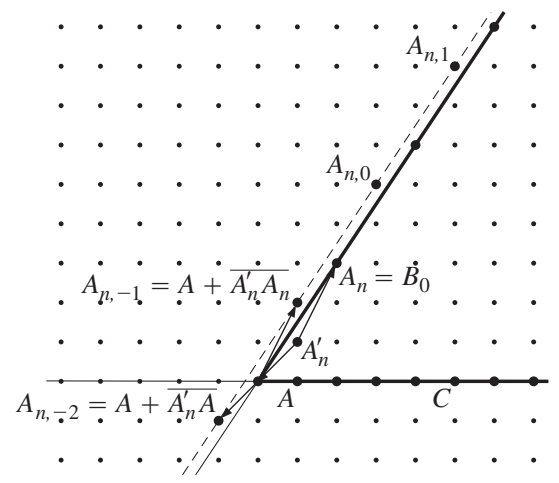

Figure 8. Lattice points $A_{n, t}$.

Since the triangle $\triangle B A D$ is $\mathscr{L}_{+}$-congruent to $\triangle A B C$, the number of points $A_{n, k}$ in the closed triangle $\triangle B A D$ is $1 \ell_{1}(A B ; C)$ : the points $A_{n, k}$ for

$$
1 \ell(A B)-1 \ell_{1}(A B ; C)-1 \leq k \leq 1 \ell(A B)-2 .
$$

Denote the integer $1 \ell(A B)-1 \ell_{1}(A B ; C)-1$ by $k_{0}$.

The point $A_{n, k_{0}}$ is contained in the segment $B_{0} B_{1}$ of the sail for the ordinary angle $\angle B A D$ (see Figure 9). Since the angles $\angle B A D$ and $\angle A B C$ are $\mathscr{L}_{+-}$ congruent, we have

$$
\begin{aligned}
t & =\operatorname{sgn}\left(A_{n-1} A A_{n}\right) \operatorname{sgn}\left(A_{n} A B_{1}\right) \operatorname{sgn}\left(A_{n-1} A_{n} B_{1}\right) 1 \sin \angle A_{n-1} A_{n} B_{1} \\
& =1 \cdot 1 \cdot \operatorname{sgn}\left(A_{n-1} A_{n} A_{n, k_{0}}\right) \sin \angle A_{n-1} A_{n} A_{n, k_{0}} \\
& =\operatorname{sign}\left(k_{0}\right)\left|k_{0}\right|=k_{0}=1 \ell(A B)-1 \ell_{1}(A B ; C)-1 .
\end{aligned}
$$

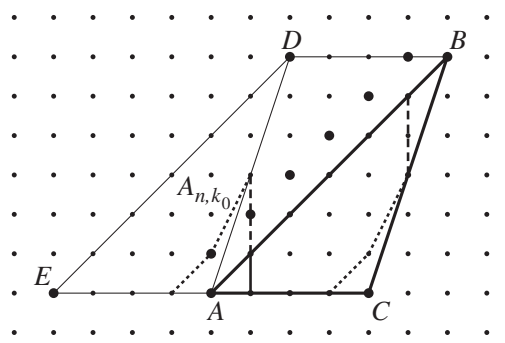

FIgURE 9. The point $A_{n, k_{0}}$.

Exactly by the same reasons,

$$
u=1 \ell(D A)-1 \ell_{1}(D A ; E)-1=1 \ell(B C)-1 \ell_{1}(B C ; A)-1 .
$$

Therefore, $\overline{L C A B}+1 \ell(A B)-1 \ell_{1}(A B ; C)-1, \overline{L A B C}+1 \ell(B C)-1 \ell_{1}(B C ; A)-1, \overline{L B C A}=$ $\pi$. 
Lemma 4.5. Let $\alpha, \beta$, and $\gamma$ be nonzero ordinary angles. Suppose that $\bar{\alpha}+{ }_{u} \bar{\beta}+{ }_{v} \bar{\gamma}=\pi$, then there exist a triangle with three consecutive ordinary angles $\mathscr{L}$-congruent to $\alpha, \beta$, and $\gamma$.

Proof. Denote by $O$ the point $(0,0)$, by $A$ the point $(1,0)$, and by $D$ the point $(-1,0)$ in the fixed system of coordinates $O X Y$.

Let us choose the points $B=\left(p_{1}, q_{1}\right)$ and $C=\left(p_{2}, q_{2}\right)$ with integers $p_{1}$, $p_{2}$ and positive integers $q_{1}, q_{2}$ such that

$$
\angle A O B=\operatorname{larctan}(1 \tan \alpha), \quad \text { and } \quad \angle A O C=\overline{\angle A O B}+{ }_{u} \bar{\beta} .
$$

Thus the vectors $\overline{O B}$ and $\overline{O C}$ defines the positive orientation, and $\angle B O C \cong \beta$. Since

$$
\bar{\alpha}+{ }_{u} \bar{\beta}+_{v} \bar{\gamma}=\pi \quad \text { and } \quad \bar{\alpha}+_{u} \bar{\beta} \cong \angle A O C
$$

the ordinary angle $\angle C O D$ is $\mathscr{L}$-congruent to $\gamma$.

Denote by $B^{\prime}$ the point $\left(p_{1} q_{2}, q_{1} q_{2}\right)$, and by $C^{\prime}$ the point $\left(p_{2} q_{1}, q_{1} q_{2}\right)$ and consider the triangle $B^{\prime} O C^{\prime}$. Since the ordinary angle $\angle B^{\prime} O C^{\prime}$ coincides with the ordinary angle $\angle B O C$, we obtain

$$
\angle B^{\prime} O C^{\prime} \cong \beta .
$$

Since the ordinary angle $\beta$ is nonzero, the points $B^{\prime}$ and $C^{\prime}$ are distinct and the straight line $B^{\prime} C^{\prime}$ does not coincide with the straight line $O A$. Since the second coordinate of the both points $B^{\prime}$ and $C^{\prime}$ equal $q_{1} q_{2}$, the straight line $B^{\prime} C^{\prime}$ is parallel to the straight line $O A$. Thus, by Proposition 1.13 it follows that

$\angle C^{\prime} B^{\prime} O \cong \angle A O B^{\prime}=\angle A O B \cong \alpha, \quad$ and $\quad \angle O C^{\prime} B^{\prime} \cong \angle C^{\prime} O D=\angle C O D \cong \gamma$

So, we have constructed the triangle $\triangle B^{\prime} O C^{\prime}$ with three consecutive ordinary angles $\mathscr{L}$-congruent to $\alpha, \beta$, and $\gamma$.

\subsection{Proof of Theorem 2.2a: conclusion of the proof}

Now we return to the proof of the first statement of the theorem on sums of lattice tangents for ordinary angles in lattice triangles.

Proof of Theorem 2.2A. Let $\alpha, \beta$, and $\gamma$ be nonzero ordinary angles satisfying the conditions i) and ii) of Theorem $2.2 \mathrm{a}$.

The second condition: $] \operatorname{ltan}(\alpha),-1, \operatorname{ltan}(\beta),-1, \operatorname{ltan}(\gamma)[=0$ implies that

$$
\bar{\alpha}+_{-1} \bar{\beta}+_{-1} \bar{\gamma}=k \pi \text {. }
$$

Since all three tangents are positive, we have $k=1$, or $k=2$. 
Consider the first condition: ]ltan $\alpha,-1,1 \tan \beta$ [ is either negative or greater than ltan $\alpha$. It implies that $\bar{\alpha}+_{-1} \bar{\beta}=0 \pi+\varphi$, for some ordinary angle $\varphi$, and hence $k=1$.

Therefore, by Lemma 4.5 there exist a triangle with three consecutive ordinary angles $\mathscr{L}$-congruent to $\alpha, \beta$, and $\gamma$.

Let us prove the converse. We prove that condition ii) of Theorem 2.2a holds by reductio ad absurdum. Suppose, that there exist a triangle $\triangle A B C$ with consecutive ordinary angles $\alpha=\angle C A B, \beta=\angle A B C$, and $\gamma=\angle B C A$, such that

$$
\left\{\begin{array}{l}
] \operatorname{ltan}(\alpha),-1, \operatorname{ltan}(\beta),-1, \operatorname{ltan}(\gamma)[\neq 0 \\
] \tan (\beta),-1, \operatorname{ltan}(\gamma),-1, \operatorname{ltan}(\alpha)[\neq 0 . \\
] \operatorname{ltan}(\gamma),-1, \operatorname{ltan}(\alpha),-1, \operatorname{ltan}(\beta)[\neq 0
\end{array}\right.
$$

These inequalities and Lemma 4.4 imply that at least two of the integers

$$
\begin{aligned}
& 1 \ell(A B)-1 \ell_{1}(A B ; C)-1, \\
& 1 \ell(B C)-1 \ell_{1}(B C ; A)-1,
\end{aligned}
$$

and

$$
1 \ell(C A)-1 \ell_{1}(C A ; B)-1
$$

are nonnegative.

Without losses of generality we suppose that

$$
\left\{\begin{array}{l}
1 \ell(A B)-1 \ell_{1}(A B ; C)-1 \geq 0 \\
1 \ell(B C)-1 \ell_{1}(B C ; A)-1 \geq 0
\end{array} .\right.
$$

Since all integers of the continued fraction

$$
\begin{aligned}
& r=] \operatorname{ltan}(\alpha), 1 \ell(A B)-1 \ell_{1}(A B ; C)-1, \\
& \qquad \operatorname{ltan}(\beta), 1 \ell(B C)-1 \ell_{1}(B C ; A)-1, \operatorname{ltan}(\gamma)[
\end{aligned}
$$

are non-negative and the last one is positive, we obtain that $r>0$ (or $r=\infty$ ). From the other hand, by Lemma 4.4 and by Theorem 4.1 we have that $r=$ $0 /-1=0$. We come to the contradiction.

Now we prove that condition i) of Theorem 2.2a holds. Suppose that there exist a triangle $\triangle A B C$ with consecutive ordinary angles $\alpha=\angle C A B, \beta=$ $\angle A B C$, and $\gamma=\angle B C A$, such that

$$
] \operatorname{ltan}(\alpha),-1, \operatorname{ltan}(\beta),-1, \operatorname{ltan}(\gamma)[=0 .
$$

Since $\bar{\alpha}+_{-1} \bar{\beta}+_{-1} \bar{\gamma}=\pi$, we have $\bar{\alpha}+_{-1} \bar{\beta}=0 \pi+\varphi$ for some ordinary angle $\varphi$. Therefore, the first condition of the theorem holds.

This concludes the proof of Theorem 2.2. 


\subsection{Theorem on sum of lattice tangents for ordinary lattice angles of convex polygons}

A satisfactory description for $\mathscr{L}$-congruence classes of lattice convex polygons has not been yet found. It is only known that the number of convex polygons with lattice area bounded from above by $n$ growths exponentially in $n^{1 / 3}$, while $n$ tends to infinity (see [2] and [3]). We conclude this section with the following theorem on necessary and sufficient condition for the lattice angles to be the angles of some convex lattice polygon.

THEOREM 4.6. Let $\alpha_{1}, \ldots, \alpha_{n}$ be an arbitrary ordered $n$-tuple of ordinary non-zero (lattice) angles. Then the following two conditions are equivalent:

- there exist a convex $n$-vertex polygon with consecutive ordinary angles $\mathscr{L}$ congruent to the ordinary angles $\alpha_{i}$ for $i=1, \ldots, n$;

- there exist a set of integers $M=\left\{m_{1}, \ldots, m_{n-1}\right\}$ such that

$$
\sum_{M, i=1}^{n} \overline{\pi-\alpha_{i}}=2 \pi .
$$

Proof. Consider an arbitrary $n$-tuple of ordinary angles $\alpha_{i}$, here $i=$ $1, \ldots, n$.

Suppose that there exist a convex polygon $A_{1} A_{2} \ldots A_{n}$ with consecutive angles $\alpha_{i}$ for $i=1, \ldots, n$. Let also the pair of vectors $\overline{A_{2} A_{3}}$ and $\overline{A_{2} A_{1}}$ defines the positive orientation of the plane (otherwise we apply to the polygon $A_{1} A_{2} \ldots A_{n}$ some $\mathscr{L}$-affine transformation changing the orientation and come to the initial position).

Let $B_{1}=O+\overline{A_{n} A_{1}}$, and $B_{i}=O+\overline{A_{i-1} A_{i}}$ for $i=2, \ldots, n$. We put by definition

$$
\beta_{i}=\left\{\begin{array}{ll}
\angle B_{i} O B_{i+1}, & \text { if } i=1, \ldots, n-1 \\
\angle B_{n} O B_{1}, & \text { if } i=n
\end{array} .\right.
$$

Consider the union of the sails for all $\beta_{i}$. This lattice oriented broken line is of the class of the extended angle with the normal form $2 \pi+0$. The LSLSsequence for this broken line contains exactly $n-1$ elements that are not contained in the LLS-sequences for the sails of $\beta_{i}$. Denote these numbers by $m_{1}, \ldots, m_{n-1}$, and the set $\left\{m_{1}, \ldots, m_{n-1}\right\}$ by $M$. Then

$$
\sum_{M, i=1}^{n} \overline{\beta_{i}}=2 \pi .
$$


From the definition of $\beta_{i}$ for $i=1, \ldots, n$ it follows that $\beta_{i} \cong \pi-\alpha_{i}$. Therefore,

$$
\sum_{M, i=1}^{n} \overline{\pi-\alpha_{i}}=2 \pi .
$$

The proof of the first part of the statement is completed.

Suppose now, that there exist a set of integers $M=\left\{m_{1}, \ldots, m_{n-1}\right\}$ such that

$$
\sum_{M, i=1}^{n} \overline{\pi-\alpha_{i}}=2 \pi .
$$

This implies that there exist lattice points $B_{1}=(1,0), B_{i}=\left(x_{i}, y_{i}\right)$, for $i=2, \ldots n-1$, and $B_{n}=(-1,0)$ such that

$$
\angle B_{i} O B_{i-1} \cong \pi-\alpha_{i-1}, \quad \text { for } i=2, \ldots, n, \quad \text { and } \quad \angle B_{1} O B_{n} \cong \pi-\alpha_{n} .
$$

Denote by $M$ the lattice point

$$
O+\sum_{i=1}^{n} \overline{O B_{i}} .
$$

Since all $\alpha_{i}$ are non-zero, the angles $\pi-\alpha_{i}$ are ordinary. Hence, the origin $O$ is an interior point of the convex hull of the points $B_{i}$ for $i=1, \ldots, k$. This implies that there exist two consecutive lattice points $B_{s}$ and $B_{s+1}$ (or $B_{n}$ and $B_{1}$ ), such that the lattice triangle $\triangle B_{s} M B_{s+1}$ contains $O$ and the edge $B_{s} B_{s+1}$ does not contain $O$. Therefore,

$$
O=\lambda_{1} \overline{O M}+\lambda_{2} \overline{O B_{i}}+\lambda_{3} \overline{O B_{i+1}},
$$

where $\lambda_{1}$ is a positive integer, and $\lambda_{2}$ and $\lambda_{3}$ are nonnegative integers. So there exist positive integers $a_{i}$, where $i=1, \ldots, n$, such that

$$
O=O+\sum_{i=1}^{n}\left(a_{i} \overline{O B_{i}}\right) .
$$

Put by definition $A_{0}=O$, and $A_{i}=A_{i-1}+a_{i} \overline{O B_{i}}$ for $i=2, \ldots, n$. The broken line $A_{0} A_{1} \ldots A_{n}$ is lattice and by the above it is closed (i. e. $A_{0}=A_{n}$ ). By construction, the ordinary angle at the vertex $A_{i}$ of the closed lattice broken line is $\mathscr{L}_{+}$-congruent to $\alpha_{i}(i=1, \ldots n)$. Since the integers $a_{i}$ are positive for $i=1, \ldots, n$ and the vectors $\overline{O B_{i}}$ are all in the counterclockwise order, the broken line is a convex polygon.

The proof of Theorem 4.6 is completed. 
REMARK 4.7. Theorem 4.6 generalizes the statement of Theorem 2.2a. Note that the direct generalization of Theorem $2.2 \mathrm{~b}$ is false: the ordinary angles do not uniquely determine the $\mathscr{L}_{+}$-affine homothety types of convex polygons. See an example on Figure 10.
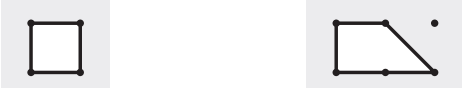

FIGURE 10. An example of different types of polygons with the $\mathscr{L}_{+}^{-}$ congruent ordinary angles.

\section{Appendix A. On global relations on algebraic singularities of complex projective toric varieties corresponding to integer-lattice triangles}

In this appendix we describe an application of theorems on sums of lattice tangents for the angles of lattice triangles and lattice convex polygons to theory of complex projective toric varieties. We refer the reader to the general definitions of theory of toric varieties to the works of V. I. Danilov [4], G. Ewald [5], W. Fulton [6], and T. Oda [18].

Let us briefly recall the definition of complex projective toric varieties associated to lattice convex polygons. Consider a lattice convex polygon $P$ with vertices $A_{0}, A_{1}, \ldots, A_{n}$. Let the intersection of this (closed) polygon with the lattice consists of the points $B_{i}=\left(x_{i}, y_{i}\right)$ for $i=0, \ldots, m$. Let also $B_{i}=A_{i}$ for $i=0, \ldots, n$. Denote by $\Omega$ the following set in $C P^{m}$ :

$$
\left\{\left(t_{1}^{x_{1}} t_{2}^{y_{1}} t_{3}^{-x_{1}-y_{1}}: t_{1}^{x_{2}} t_{2}^{y_{2}} t_{3}^{-x_{2}-y_{2}}: \ldots: t_{1}^{x_{m}} t_{2}^{y_{m}} t_{3}^{-x_{m}-y_{m}}\right) \mid t_{1}, t_{2}, t_{3} \in \mathrm{C} \backslash\{0\}\right\} .
$$

The closure of the set $\Omega$ in the natural topology of $C P^{m}$ is called the complex toric variety associated with the polygon $P$ and denoted by $X_{P}$.

For any $i=0, \ldots, m$ we denote by $\tilde{A}_{i}$ the point $(0: \ldots: 0: 1: 0: \ldots: 0)$ where 1 stands on the $(i+1)$-th place.

From general theory it follows that:

a) the set $X_{P}$ is a complex projective complex-two-dimensional variety with isolated algebraic singularities;

b) the complex toric projective variety contains the points $\tilde{A}_{i}$ for $i=$ $0, \ldots, n$ (where $n+1$ is the number of vertices of convex polygon);

c) the points of $X_{P} \backslash\left\{\tilde{A}_{0}, \tilde{A}_{1}, \ldots, \tilde{A}_{n}\right\}$ are non-singular;

d) the point $\tilde{A}_{i}$ for any integer $i$ satisfying $0 \leq i \leq n$ is singular iff the corresponding ordinary angle $\alpha_{i}$ at the vertex $A_{i}$ of the polygon $P$ is not $\mathscr{L}$-congruent to $\operatorname{larctan}(1)$; 
e) the algebraic singularity at $\tilde{A}_{i}$ for any integer $i$ satisfying $0 \leq i \leq n$ is uniquely determined by the $\mathscr{L}$-affine type of the non-oriented sail of the lattice angle $\alpha_{i}$.

The algebraic singularity is said to be toric if there exists a projective toric variety with the given algebraic singularity.

Note that the $\mathscr{L}$-affine classes of non-oriented sails for angles $\alpha$ and $\beta$ coincide iff $\beta \cong \alpha$, or $\beta \cong \alpha^{t}$. This allows us to associate to any complex-twodimensional toric algebraic singularity, corresponding to the sail of the angle $\alpha$, the unordered pair of rationals $(a, b)$, where $a=\operatorname{ltan} \alpha$ and $b=\operatorname{ltan} \alpha^{t}$.

Remark A.1. Note that the continued fraction for the sail $\alpha$ is slightly different to the Hirzebruch-Jung continued fractions for toric singularities (see the works [9] by H. W. E. Jung, and [8] by F. Hirzebruch). The relations between these continued fractions is described in the paper [19] by P. Popescu-Pampu.

Corollary A.2. Suppose, that we are given by three complex-two-dimensional toric singularities defined by pairs of rationals $\left(a_{i}, b_{i}\right)$ for $i=1,2,3$. There exist a complex toric variety associated with some triangle with these three singularities iff there exist a permutation $\sigma \in S_{3}$ and the rationals $c_{i}$ from the sets $\left\{a_{i}, b_{i}\right\}$ for $i=1,2,3$, such that the following conditions hold:

i) the continued fraction $] c_{\sigma(1)},-1, c_{\sigma(2)}[$ is either negative, or greater than $c_{\sigma(1)}$, or equals $\infty$;

ii) $] c_{\sigma(1)},-1, c_{\sigma(2)},-1, c_{\sigma(3)}[=0$.

We note again that we use odd continued fractions for $c_{1}, c_{2}$, and $c_{3}$ in the statement of the above proposition (see Subsection 2.1 for the notation of continued fractions).

Proof. The proposition follows directly from Theorem 2.2a.

Proposition A.3. For any collection (with multiplicities) of complex-twodimensional toric algebraic singularities there exist a complex-two-dimensional toric projective variety with exactly the given collection of toric singularities.

For the proof of Proposition A.3 we need the following lemma.

Lemma A.4. For any collection of ordinary angles $\alpha_{i}(i=1, \ldots, n)$, there exist an integer $k \geq n-1$ and a $k$-tuple of integers $M=\left(m_{1}, \ldots, m_{k}\right)$, such that

$$
\overline{\alpha_{1}}+_{m_{1}} \cdots+_{m_{n-1}} \overline{\alpha_{n}}+_{m_{n}} \operatorname{larctan}(1)+_{m_{n+1}} \cdots+_{m_{k}} \operatorname{larctan}(1)=2 \pi .
$$

Proof. Consider any collection of ordinary angles $\alpha_{i}(i=1, \ldots, n)$ and denote

$$
\Phi=\overline{\alpha_{1}}+{ }_{1} \overline{\alpha_{2}}+{ }_{1} \cdots+{ }_{1} \overline{\alpha_{n}} .
$$


There exist an oriented lattice broken line for the angle $\Phi$ with the LSLSsequence with positive elements. Hence, $\Phi \hat{\cong} \varphi+0 \pi$.

If $\varphi \cong \operatorname{larctan}(1)$, we have

$$
\Phi+{ }_{-2} \operatorname{larctan}(1)+_{-2} \operatorname{larctan}(1)+_{-2} \operatorname{larctan}(1)=2 \pi .
$$

Then $k=n+2$, and $M=(1, \ldots, 1,-2,-2,-2)$.

Suppose now $\varphi \nsucceq \operatorname{larctan}(1)$, then the following holds

$$
\bar{\varphi}+_{-1} \overline{\pi-\varphi}+_{-2} \operatorname{larctan}(1)+_{-2} \operatorname{larctan}(1)=2 \pi .
$$

Consider the sail for the angle $\pi-\varphi$. Suppose the sequence of all its lattice points (not only vertices) is $B_{0}, \ldots, B_{s}$ (with the order coinciding with the order of the sail). Then we have

$$
\angle B_{i} O B_{i+1} \hat{\cong} \operatorname{larctan}(1) \quad \text { for any } \quad i=1, \ldots, s .
$$

Denote by $b_{i}$ the values of $1 \sin \angle B_{i} O B_{i+1}$ for $i=1, \ldots, s$. Then we have

$$
\begin{aligned}
\bar{\varphi}++_{-2} & \operatorname{larctan}(1)+_{-2} \operatorname{larctan}(1)++_{-2} \operatorname{larctan}(1) \\
= & \overline{\alpha_{1}}+{ }_{1} \overline{\alpha_{2}}+{ }_{1} \cdots+_{1} \overline{\alpha_{n}}+{ }_{-1} \operatorname{larctan}(1)+_{b_{1}} \operatorname{larctan}(1)+_{b_{2}} \\
& \quad \cdots+_{b_{s}} \operatorname{larctan}(1)+_{-2} \operatorname{larctan}(1)+_{-2} \operatorname{larctan}(1)+_{-2} \operatorname{larctan}(1) \\
= & 2 \pi .
\end{aligned}
$$

Therefore, $k=n+s+3$, and

$$
M=(\underbrace{1,1, \ldots, 1,1}_{(n-1) \text {-times }},-1, b_{1}, \ldots, b_{s},-2,-2,-2) .
$$

The proof of Lemma A.4 is completed.

Proof of the statement of the Proposition A.3. Consider an arbitrary collection of two-dimensional toric algebraic singularities. Suppose that they are represented by ordinary angles $\alpha_{i}(i=1, \ldots, n)$. By Lemma A.4 there exist an integer $k \geq n-1$ and a k-tuple of integers $M=\left(m_{1}, \ldots, m_{k}\right)$, such that

$\overline{\left(\pi-\alpha_{1}\right)}+{ }_{m_{1}} \cdots+{ }_{m_{n-1}} \overline{\left(\pi-\alpha_{n}\right)}+{ }_{m_{n}} \operatorname{larctan}(1)+{ }_{m_{n+1}} \cdots+{ }_{m_{k}} \operatorname{larctan}(1)=2 \pi$.

By Theorem 4.6 there exist a convex polygon $P=A_{0} \ldots A_{k}$ with angles $\mathscr{L}_{+}$-congruent to the ordinary angles $\alpha_{i}(i=1, \ldots, n)$, and $k-n+1$ angles $\operatorname{larctan}(1)$.

By the above, the toric variety $X_{P}$ is nonsingular at points of $P_{X} \backslash\left\{\tilde{A}_{0}, \tilde{A}_{1}\right.$, $\left.\ldots, \tilde{A}_{k}\right\}$. It is also nonsingular at the points $\tilde{A}_{i}$ with the corresponding ordinary 
angles $\mathscr{L}$-congruent to $\operatorname{larctan}(1)$. The collection of the toric singularities at the remaining points coincide with the given collection.

This concludes the proof of Proposition A.3.

On Figure 11 we show an example of the polygon for a projective toric variety with the unique toric singularity, represented by the sail of $\operatorname{larctan}(7 / 5)$. The ordinary angle $\alpha$ on the figure is $\mathscr{L}_{+}$-congruent to $\operatorname{larctan}(7 / 5)$, the angles $\beta$ and $\gamma$ are $\mathscr{L}_{+}$-congruent to $\operatorname{larctan}(1)$.
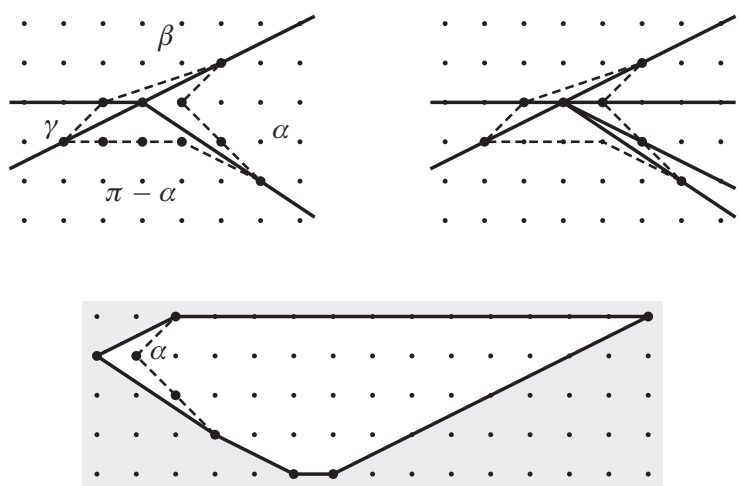

FIGURE 11. Constructing a polygon with all angles $\mathscr{L}_{+}$-congruent to $\operatorname{larctan}(1)$ except one angle that is $\mathscr{L}_{+}$-congruent to $\operatorname{larctan}(7 / 5)$.

\section{Appendix B. On $\mathscr{L}$-congruence criterions for lattice triangles}

Here we discuss the $\mathscr{L}$-congruence criterions for lattice lattice triangles. By the first criterion of $\mathscr{L}$-congruence for lattice triangles we obtain that the number of $\mathscr{L}$-congruence classes for lattice triangles with bounded lattice area is finite. We write down the numbers of $\mathscr{L}$-congruence classes for triangles with lattice area less then or equal to 20 .

On criterions of lattice triangle $\mathscr{L}$-congruence. We start with the study of lattice analogs for the first, the second, and the third Euclidean criterions of triangle congruence.

Statement B.1 (The first criterion of lattice triangle $\mathscr{L}$-congruence). Consider two lattice triangles $\triangle A B C$ and $\triangle A^{\prime} B^{\prime} C^{\prime}$. Suppose that the edge $A B$ is $\mathscr{L}$-congruent to the edge $A^{\prime} B^{\prime}$, the edge $A C$ is $\mathscr{L}$-congruent to the edge $A^{\prime} C^{\prime}$, and the ordinary angle $\angle C A B$ is $\mathscr{L}$-congruent to the ordinary angle $\angle C^{\prime} A^{\prime} B^{\prime}$, then the triangle $\triangle A^{\prime} B^{\prime} C^{\prime}$ is $\mathscr{L}$-congruent to the $\triangle A B C$.

It turns out that the second and the third criterions taken from Euclidean geometry do not hold. The following two examples illustrate these phenomena. 
Example B.2. The second criterion of triangle $\mathscr{L}$-congruence does not hold in lattice geometry. On Figure 12 we show two lattice triangles $\triangle A B C$ and $\triangle A^{\prime} B^{\prime} C^{\prime}$. The edge $A B$ is $\mathscr{L}$-congruent to the edge $A^{\prime} B^{\prime}$ (here $1 \ell\left(A^{\prime} B^{\prime}\right)=$ $1 \ell(A B)=4)$. The ordinary angle $\angle A B C$ is $\mathscr{L}$-congruent to the ordinary angle $\angle A^{\prime} B^{\prime} C^{\prime}$ (since $\angle A B C \cong \angle A^{\prime} B^{\prime} C^{\prime} \cong \operatorname{larctan}(1)$ ), and the ordinary angle $\angle C A B$ is $\mathscr{L}$-congruent to the ordinary angle $\angle C^{\prime} A^{\prime} B^{\prime}$ (since $\angle C A B \cong$ $\angle C^{\prime} A^{\prime} B^{\prime} \cong$ larctan(1)), The triangle $\triangle A^{\prime} B^{\prime} C^{\prime}$ is not $\mathscr{L}$-congruent to the triangle $\triangle A B C$, since $\operatorname{lS}(\triangle A B C)=4$ and $\operatorname{lS}\left(\triangle A^{\prime} B^{\prime} C^{\prime}\right)=8$.
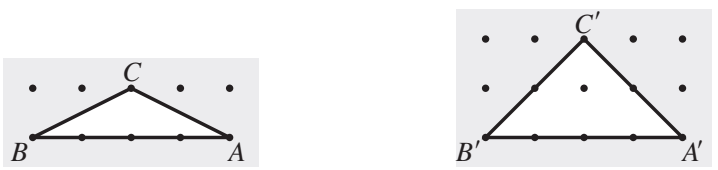

FigURE 12. The second criterion of triangle $\mathscr{L}$-congruence does not hold.

ExAmple B.3. The third criterion of triangle $\mathscr{L}$-congruence does not hold in lattice geometry. On Figure 13 we show two lattice triangles $\triangle A B C$ and $\triangle A^{\prime} B^{\prime} C^{\prime}$. All edges of both triangles are $\mathscr{L}$-congruent (of length one), but the triangles are not $\mathscr{L}$-congruent, since $\operatorname{lS}(\triangle A B C)=1$ and $1 \mathrm{~S}\left(\triangle A^{\prime} B^{\prime} C^{\prime}\right)=3$.
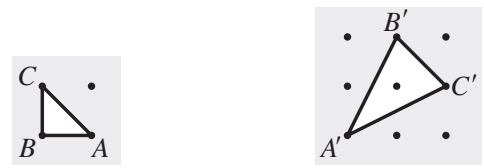

FIGURE 13. The third criterion of triangle $\mathscr{L}$-congruence does not hold.

Instead of the second and the third criterions there exists the following additional criterion of lattice triangles $\mathscr{L}$-congruence.

Statement B.4 (An additional criterion of lattice triangle integer-congruence). Consider two lattice triangles $\triangle A B C$ and $\triangle A^{\prime} B^{\prime} C^{\prime}$ of the same lattice area. Suppose that the ordinary angle $\angle A B C$ is $\mathscr{L}$-congruent to the ordinary angle $\angle A^{\prime} B^{\prime} C^{\prime}$, the ordinary angle $\angle C A B$ is $\mathscr{L}$-congruent to the ordinary angle $\angle C^{\prime} A^{\prime} B^{\prime}$, the ordinary angle $\angle B C A$ is $\mathscr{L}$-congruent to the ordinary angle $\angle B^{\prime} C^{\prime} A^{\prime}$, then the triangle $\triangle A^{\prime} B^{\prime} C^{\prime}$ is $\mathscr{L}$-congruent to the triangle $\triangle A B C$.

In the following example we show that the additional criterion of lattice triangle $\mathscr{L}$-congruence is not improvable.

Example B.5. On Figure 14 we show an example of two lattice nonequivalent triangles $\triangle A B C$ and $\triangle A^{\prime} B^{\prime} C^{\prime}$ of the same lattice area equals 4 
and the same ordinary angles $\angle A B C, \angle C A B$, and $\angle A^{\prime} B^{\prime} C^{\prime}, \angle C^{\prime} A^{\prime} B^{\prime}$ all $\mathscr{L}$ equivalent to the angle larctan(1), but $\triangle A B C \mp \triangle A^{\prime} B^{\prime} C^{\prime}$.
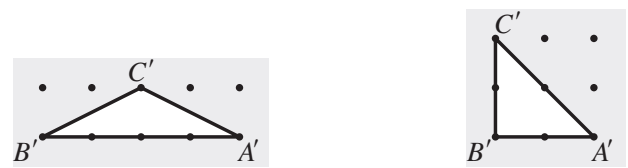

FIGURE 14 . The additional criterion of lattice triangle $\mathscr{L}$-congruence is not improvable.

Lattice triangles of small area. The above criterions allows to enumerate all lattice triangles of small lattice area up to the lattice equivalence. In the following table we write down the numbers $N(d)$ of nonequivalent lattice triangles of lattice area $d$ for $d \leq 20$.

\begin{tabular}{|c|c|c|c|c|c|c|c|c|c|c|c|c|c|c|c|c|c|c|c|c|c|}
\hline$d$ & 1 & 2 & 3 & 4 & \begin{tabular}{l|}
5 \\
\end{tabular} & 6 & 78 & c & 1 & & 11 & 12 & 13 & 14 & 15 & 16 & 17 & 18 & \begin{tabular}{l|l}
8 & 1
\end{tabular} & & \\
\hline$N(d)$ & 1 & 1 & 2 & 3 & 2 & 4 & 45 & 55 & 6 & & 4 & 10 & 6 & 8 & 8 & 11 & 6 & 13 & 38 & & 14 \\
\hline
\end{tabular}

As it is easy to show, we always have $d / 3 \leq N(d) \leq d$. The asymptotic behaviour of $N(d)$ and even of the average of $N(d)$ (if they exist) is unknown to the author.

\section{Appendix C. Some unsolved question on lattice trigonometry}

We conclude this paper with a small collection of unsolved questions.

Let us start with some questions on elementary definitions of lattice trigonometry. In this paper we do not show any geometrical meaning of lattice cosine. Here arise the following question.

Problem 1. Find a natural description of lattice cosine for ordinary angles in terms of lattice invariants of the corresponding sublattices.

This problem seems to be close to the following one.

Problem 2. Does there exist a lattice analog of the cosine formula for the angles of triangles in Euclidean geometry?

Let us continue with questions on lattice analogs of classical trigonometric formulas for trigonometric functions of angles of triangles in Euclidean geometry.

Problem 3. a) Knowing the lattice trigonometric functions for lattice angles $\alpha, \beta$ and integer $n$, find the explicit formula for the lattice trigonometric functions of the extended angle $\bar{\alpha}+{ }_{n} \bar{\beta}$. 
b) Knowing the lattice trigonometric functions for a lattice angle $\alpha$, an integer $m$, and positive integer $m$, find the explicit formula for the lattice trigonometric functions of the extended angle

$$
\sum_{M, i=1}^{l} \bar{\alpha}
$$

where $M=(m, \ldots, m)$ is an $n$-tuple.

Now we formulate a problem on generalization of the statement of Theorem $2.2 \mathrm{~b}$ to the case of $n$ ordinary angles. Such generalization is important in toric geometry and theory of multidimensional continued fractions.

Problem 4. Find a necessary and sufficient conditions for the existence of an $n$-gon with the given ordered sequence of ordinary angles $\left(\alpha_{1}, \ldots, \alpha_{n}\right)$ and the consistent sequence of lattice lengths of the edges $\left(l_{1}, \ldots, l_{n}\right)$ in terms of continued fractions for $n \geq 4$.

We conclude this paper with the following problem. We remind that $(N(d))$ is the numbers of nonequivalent lattice triangles having the lattice area being equal to $d$ (see Appendix B).

Problem 5. Find an explicit formula for the numbers $N(d)$.

\section{REFERENCES}

1. Arnold, V. I., Continued fractions, M., Moscow Center of Continuous Mathematical Education, 2002.

2. Arnold, V. I., Statistics of integer convex polygons, Funct. Anal. Appl. 14(2) (1980), 1-3.

3. Bárány, I., Vershik, A. M., On the number of convex lattice polytopes, Geom. Funct. Anal. 2(4) (1992), 381-393.

4. Danilov, V. I., The geometry of toric varieties, Uspekhi Mat. Nauk. 33(2) (1978), 85-134.

5. Ewald, G., Combinatorial Convexity and Algebraic Geometry, Grad. Texts in Math. 168 (1996).

6. Fulton, W., Introduction to Toric Varieties, Ann. of Math. Stud. 131 (1993).

7. Hinchin, A. Ya., Continued Fractions, M.: FISMATGIS, 1961.

8. Hirzebruch, F., Über vierdimensionale Riemannsche Flächen Mehrdeutinger analystischer Funktionen von zwei komplexen Veränderlichen, Math. Ann. 126 (1953), 1-22.

9. Jung, H. W. E., Darstellung der Funktionen eines algebraischen Körpsers zweier unabhängigen Veränderlichen $x, y$ in der Umgebung einer Stelle $x=a, y=b$, J. Reine Angew. Math. 133 (1908), 289-314.

10. Karpenkov, O., On tori decompositions associated with two-dimensional continued fractions of cubic irrationalities, Funct. Anal. Appl. 38(2) (2004), 28-37.

11. Karpenkov, O., Classification of lattice-regular lattice convex polytopes, http://arxiv.org/abs/math.CO/0602193. 
12. Khovanskii, A. G., Pukhlikov, A., Finitely additive measures of virtual polytopes, Algebra and Analysis, 4(2) (1992), 161-185; translation in St. Petersburg Math. J. 4(2) (1993), 337-356.

13. Khovanskii, A. G., Pukhlikov, A., A Riemann-Roch theorem for integrals and sums of quasipolynomials over virtual polytopes, Algebra and Analysis 4(4) (1992), 188-216; translation in St. Petersburg Math. J. 4(4) (1993), 789-812.

14. Klein, F., Ueber einegeometrische Auffassung der gewöhnliche Kettenbruchentwicklung, Nachr. Ges. Wiss. Göttingen Math-Phys. K1. 3 (1891), 357-359.

15. Kontsevich, M. L., and Suhov, Yu. M., Statistics of Klein Polyhedra and Multidimensional Continued Fractions, Amer. Math. Soc. Transl. 197(2) (1999), 9-27.

16. Korkina, E. I., Two-dimensional continued fractions. The simplest examples, Proc. Steklov Inst. Math. 209 (1995), 243-166.

17. Lachaud, G., Voiles et Polyèdres de Klein, preprint n 95-22, Laboratoire de Mathématiques Discrètes du C.N.R.S., Luminy (1995).

18. Oda, T., Convex bodies and Algebraic Geometry, An Introduction to the Theory of Toric Varieties, Ergeb. Math. Grenzgeb. 145 (1988)

19. Popescu-Pampu, P., The Geometry of Continued Fractions and The Topology of Surface Singularities, ArXiv:math.GT/0506432, v1 (2005).

CEREMADE - UMR 7534

UNIVERSITÉ PARIS-DAUPHINE FRANCE

75775 PARIS CEDEX 16

FRANCE

E-mail: karpenk@mccme.ru 